\title{
Data assimilation for moving mesh methods with an application to ice sheet modelling
}

\author{
Bertrand Bonan, Nancy K. Nichols, Michael J. Baines, and Dale Partridge \\ School of Mathematical, Physical and Computational Sciences, University of Reading, Reading, UK \\ Correspondence to: Nancy K. Nichols (n.k.nichols@reading.ac.uk)
}

Received: 22 August 2016 - Discussion started: 12 September 2016

Revised: 19 July 2017 - Accepted: 25 July 2017 - Published: 5 September 2017

\begin{abstract}
We develop data assimilation techniques for nonlinear dynamical systems modelled by moving mesh methods. Such techniques are valuable for explicitly tracking interfaces and boundaries in evolving systems. The unique aspect of these assimilation techniques is that both the states of the system and the positions of the mesh points are updated simultaneously using physical observations. Covariances between states and mesh points are generated either by a correlation structure function in a variational context or by ensemble methods. The application of the techniques is demonstrated on a one-dimensional model of a grounded shallow ice sheet. It is shown, using observations of surface elevation and/or surface ice velocities, that the techniques predict the evolution of the ice sheet margin and the ice thickness accurately and efficiently. This approach also allows the straightforward assimilation of observations of the position of the ice sheet margin.
\end{abstract}

\section{Introduction}

From lava flows to tumour growth to water flooding, many time-evolving processes can be mathematically modelled as moving boundary problems. Predicting their evolution accurately requires not only the estimation of the state variables of the system over a moving domain but also the estimation of the location of the moving domain itself. In this paper, we propose to combine data assimilation with a moving mesh numerical model to estimate both the domain and the states of a moving boundary problem. Genuine moving mesh methods use a fixed number of mesh points whose movement can be generated by various techniques (Budd et al., 2009; Baines et al., 2011). The moving mesh method used here is based on conservation of local mass fractions (Baines et al., 2005, 2011; Partridge, 2013; Lee et al., 2015; Sarahs, 2016). The major advantage of our moving mesh method is that only a small number of mesh steps are needed to accurately determine the positions of the boundaries, unlike fixed or adaptive mesh methods (Berger and Oliger, 1984; Li et al., 2014; Cornford et al., 2013, 2016; Gladstone et al., 2010). Our moving mesh method has been successfully applied to a number of moving boundary problems, including one- and two-dimensional models of ice sheet flow, tumour growth and chemical spreading (Partridge, 2013; Bonan et al., 2016; Lukyanov et al., 2012; Lee et al., 2013).

Data assimilation (DA) aims to combine available observations of a dynamical system with model predictions in order to provide optimal estimates of the state of the system and an estimation of the uncertainty in these estimates. DA has been applied successfully in various contexts and is routinely used in operational systems such as numerical weather prediction systems (Lahoz et al., 2010; Blayo et al., 2014). In particular, DA has already been used with fixed and adaptive grid models in the context of moving boundary problems. In these cases, estimates outside the moving domain are generally non-physical and need to be reanalysed (Mathiot et al., 2012; Bonan et al., 2014). Furthermore, with fixed or adaptive grids, DA does not provide an explicit estimate of the extent of the domain; this can be only done by interpolation. By combining DA with our moving mesh numerical model, we show here that the explicit extent of the domain can be estimated efficiently and accurately and that non-physical estimates do not appear.

Our approach is particularly relevant to the prediction of the dynamics of ice sheets and glaciers. Future evolution of ice sheet boundaries is closely linked with sea level rise 
(Church et al., 2013) and ice sheets are now relatively wellobserved bodies (Vaughan et al., 2013). Our moving mesh numerical method for ice flow has already been validated for both 1-D and 2-D models of ice sheets (Partridge, 2013; Bonan et al., 2016). In this paper, we describe the application of data assimilation to the moving mesh method and demonstrate the combined techniques using a one-dimensional moving mesh model of a grounded shallow ice sheet as described in Bonan et al. (2016). Although the model is relatively simple, there is no reason that these techniques cannot be extended to much more complex problems.

We adapt here two popular DA schemes, a 3-D variational scheme (or 3D-Var; see, e.g. Lorenc, 1986; Nichols, 2010) and an ensemble transform Kalman filter (ETKF; see Bishop et al., 2001; Hunt et al., 2007), to estimate the state of an ice sheet modelled by our moving mesh method (Bonan et al., 2016). The approach is validated by twin experiments using available classical surface observations (surface elevation and surface velocity; see Vaughan et al., 2013). Observations of the position of the moving boundary (see, e.g. Dyke and Prest, 1987 for observations of continental margins in palaeoglaciology) are also assimilated using a straightforward observation operator. The paper is organised as follows: in Sect. 2 we recall the key points of the moving point ice sheet model, in Sect. 3 we describe how to apply the 3D-Var and the ETKF methods for our state estimation problem and in Sects. 4 and 5 we validate our approach by performing several twin experiments before concluding in Sect. 6 .

\section{Moving point ice sheet model}

\subsection{Ice sheet dynamics}

We consider a single-phase, radially symmetric, grounded ice sheet (no floating ice), centred on the origin $r=0$ of the radial coordinates. The origin is called the ice divide.

The geometry of the grounded ice sheet is described by its surface altitude, $s(t, r)$, the ice thickness, $h(t, r)$ and the altitude, $b(r)$, of the fixed bedrock on which the ice sheet lies (see Fig. 1). These quantities are linked through the relation

$s=b+h$.

The position of the edge of the ice sheet $r_{l}(t)$, also known as the ice sheet margin, is implicitly determined by the Dirichlet boundary condition

$h\left(t, r_{l}(t)\right)=0$.

The evolution of an ice sheet is governed by the balance between the mass exchanges at the surface (snow precipitation and surface melting) and the ice flow that carries the ice from the interior of the ice sheet towards its margins. This is summarised by the mass balance equation

$$
\frac{\partial h}{\partial t}=m(t, r)-\frac{1}{r} \frac{\partial(r h U)}{\partial r},
$$

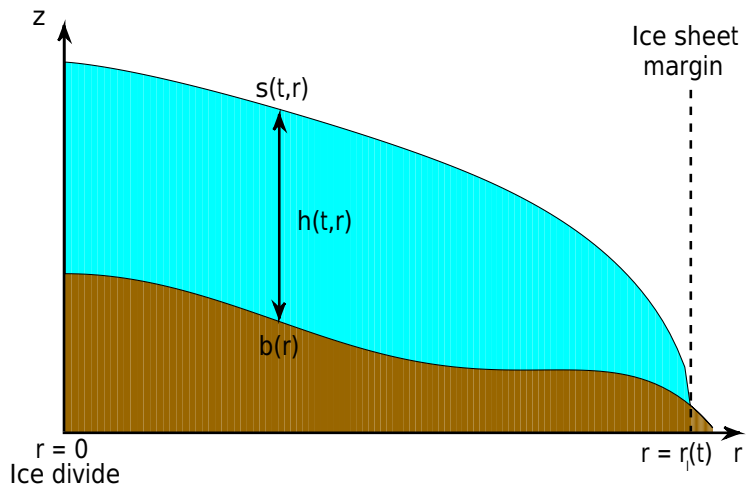

Figure 1. Section of a grounded radially symmetrical ice sheet.

Table 1. Parameters involved in the computation of the vertically averaged horizontal component of the ice velocity (Eq. 4).

\begin{tabular}{lll}
\hline Parameter & & Value \\
\hline$n$ & exponent of the creep relation & 3 \\
$A$ & coefficient of the creep relation & $10^{-16} \mathrm{~Pa}^{-3} \mathrm{yr}^{-1}$ \\
$\rho_{\mathrm{i}}$ & density of ice & $910 \mathrm{~kg} \mathrm{~m}^{-3}$ \\
$g$ & gravitational acceleration & $9.81 \mathrm{~m} \mathrm{~s}^{-2}$ \\
\hline
\end{tabular}

where $m(t, r)$ is the surface mass balance and $U(t, r)$ is the vertically averaged horizontal component of the ice velocity in the sheet. In the numerical experiments (see Sects. 4 and 5), we use two different surface mass balances: a function that only depends on the radius $r$ and a more complex surface mass balance which depends on the atmospheric temperature that evolves with the geometry of the ice sheet. Both surface mass balances are described in detail in Appendix A.

The velocity of the ice is derived using the shallow ice approximation (Hutter, 1983), which leads to the following analytical formulation of the vertically averaged horizontal component of the ice velocity $U(t, r)$ :

$U=-\frac{2}{n+2} A\left(\rho_{\mathrm{i}} g\right)^{n} h^{n+1}\left|\frac{\partial s}{\partial r}\right|^{n-1} \frac{\partial s}{\partial r}$,

where $s$ is given by Eq. (1) and the parameters involved in the shallow ice approximation (SIA) are summarised in Table 1 . Since we consider only radially symmetrical ice sheets, a symmetry condition also holds at $r=0$

$U(t, 0)=0$ and $\frac{\partial s}{\partial r}(t, 0)=0$.

\subsection{Moving point method}

The moving point numerical method we use in this paper relies on the computation of point velocities and point locations. This type of method belongs to the family of velocitybased (or Lagrangian) methods (Cao et al., 2003). Here, the 
velocity of mesh points is obtained by conserving local mass fractions (Baines et al., 2005, 2011). To calculate the velocity, we first define the total volume of the ice sheet $\theta(t)$ as

$\theta(t)=2 \pi \int_{0}^{r_{l}(t)} r h(t, r) \mathrm{d} r$.

Assuming that the flux of ice through the ice sheet margin is zero, its rate of change $\dot{\theta}$ depends only on the surface mass balance,

$\dot{\theta}(t)=2 \pi \int_{0}^{r_{l}(t)} r m(t, r) \mathrm{d} r$.

We now define the relative mass fraction $\mu(\hat{r})$ relative to the moving point $\hat{r}(t)$. Since the density of ice $\rho_{\mathrm{i}}$ is assumed constant, volume fractions and mass fractions are equivalent and

$\mu(\hat{r})=\frac{2 \pi}{\theta(t)} \int_{0}^{\hat{r}(t)} r h(t, r) \mathrm{d} r$.

The velocity of the moving point $\hat{r}(t)$ is defined implicitly by keeping $\mu(\hat{r})$ constant in time, that is, $\frac{d \mu(\hat{r})}{\mathrm{d} t}=0$. By differentiating Eq. (8) with respect to time using the Leibniz integral rule, we obtain the velocity of every interior point

$$
\begin{aligned}
\frac{\mathrm{d} \hat{r}}{\mathrm{~d} t} & =U(t, \hat{r}(t))+\frac{1}{\hat{r}(t) h(t, \hat{r}(t))} \\
& \left(\mu(\hat{r}) \int_{0}^{r_{l}(t)} r m(t, r) \mathrm{d} r-\int_{0}^{\hat{r}(t)} r m(t, r) \mathrm{d} r\right) .
\end{aligned}
$$

One of the points is dedicated to the static ice divide at $r=0$, while another point tracks the position of the margin $r_{l}(t)$, which moves at the velocity (Bonan et al., 2016)

$\frac{\mathrm{d} r_{l}}{\mathrm{~d} t}=U\left(t, r_{l}(t)\right)-m\left(t, r_{l}(t)\right)\left(\frac{\partial h}{\partial r}\right)^{-1}$.

Once the velocity of each moving point has been obtained from Eq. (9) or (10), the moving points are moved in a Lagrangian manner using the explicit Euler scheme:

$\hat{r}(t+\Delta t)=\hat{r}(t)+\Delta t \frac{\mathrm{d} \hat{r}}{\mathrm{~d} t}$.

The total mass $\theta(t)$ is updated in the same way using $\dot{\theta}(t)$ from Eq. (7). Finally, the ice thickness profile is updated by differentiating Eq. (8) with respect to $\hat{r}$, giving

$h(t, \hat{r}(t))=\frac{\theta(t)}{\pi} \frac{\mathrm{d} \mu(\hat{r})}{\mathrm{d}\left(\hat{r}^{2}\right)}$.

\subsection{Numerical model}

From the equations detailed in Sect. 2.2, a finite difference algorithm is derived (see Bonan et al., 2016 for the full algorithm). The mesh consists of $n_{r}$ moving nodes with the positions

$0=\hat{r}_{1}<\hat{r}_{2}<\ldots<\hat{r}_{n_{r}-1}<\hat{r}_{n_{r}}=r_{l}(t)$.

No further assumption is made on the spatial distribution of the moving nodes. At each node $\hat{r}_{i}$ there is an associated ice thickness $h_{i}$ and a fixed mass fraction $\mu_{i}$. By construction, $\mu_{1}=0, \mu_{n_{r}}=1$ and the ice thickness at the ice sheet margin $h_{n_{r}}=0$.

The user provides the initial mesh and the ice thickness at mesh points in order to initialise the numerical model. From these quantities, the total mass and the mass fractions at the initial time are calculated by discretising Eqs. (6) and (8) using the following composite trapezoidal rule:

$$
\begin{aligned}
\theta & =\frac{\pi}{2} \sum_{i=1}^{n_{r}-1}\left(h_{i}+h_{i+1}\right)\left(\hat{r}_{i+1}^{2}-\hat{r}_{i}^{2}\right), \\
\mu_{1} & =0, \mu_{i+1}=\mu_{i}+\frac{\pi}{2 \theta}\left(h_{i}+h_{i+1}\right)\left(\hat{r}_{i+1}^{2}-\hat{r}_{i}^{2}\right), \\
i & =1, \ldots, n_{r}-1 .
\end{aligned}
$$

The mesh points are then evolved using a discrete form of Eq. (9) and the ice thickness is determined using a discrete form of Eq. (12), with the mass fractions $\left\{\mu_{i}\right\}$ kept constant over a time step. Full details are given in Bonan et al. (2016).

\section{State estimation of a system modelled with a moving mesh}

We now recall the basics of data assimilation before explaining how to adapt the 3D-Var and the ETKF methods to our context. We then clarify the form of the observation operator for various types of observations that we assimilate.

\subsection{Data assimilation}

We consider data assimilation in a discrete dynamical system evolving in time. We denote by $\boldsymbol{x}_{k}$ the vector of size $n_{\boldsymbol{x}}$ describing the state of the system at time $t_{k}$. For example, in our numerical ice sheet model, ice thicknesses at mesh points are elements of the state vector. The state $\boldsymbol{x}_{k}$ is propagated forward in time to a time $t_{k+1}$ by the non-linear model $\mathcal{M}_{k, k+1}$. Assuming the model is perfect, we have

$\boldsymbol{x}_{k+1}=\mathcal{M}_{k, k+1}\left(\boldsymbol{x}_{k}\right)$.

Observations are available at times $t_{k}$ and are related to $\boldsymbol{x}_{k}$ through the equation

$\boldsymbol{y}_{k}=\mathcal{H}_{k}\left(\boldsymbol{x}_{k}\right)+\boldsymbol{\varepsilon}_{k}$, 
where $\boldsymbol{y}_{k}$ is a vector of $p_{k}$ observations taken at time $t_{k}, \mathcal{H}_{k}$ is the (possibly non-linear) observation operator and $\boldsymbol{\varepsilon}_{k}$ is the observation error vector, which is assumed to be unbiased (zero mean) with covariance matrix $\mathbf{R}_{k}$.

The objective of DA is to provide an optimal estimate $\boldsymbol{x}_{k}^{a}$ of the system, called the analysis, by combining observations with information derived from the model. We consider in this paper two different DA schemes: a 3D-Var scheme and an ETKF.

\subsubsection{D-Var}

The 3D-Var method (see, e.g. Lorenc, 1986; Nichols, 2010) aims to provide the optimal estimate $\boldsymbol{x}_{k}^{a}$ by minimising the cost function

$$
\begin{aligned}
\mathcal{J}(\boldsymbol{x}) & =\frac{1}{2}\left(\boldsymbol{x}-\boldsymbol{x}_{k}^{b}\right)^{T} \mathbf{B}_{k}^{-1}\left(\boldsymbol{x}-\boldsymbol{x}_{k}^{b}\right) \\
& +\frac{1}{2}\left(\boldsymbol{y}_{k}-\mathcal{H}_{k}(\boldsymbol{x})\right)^{T} \mathbf{R}_{k}^{-1}\left(\boldsymbol{y}_{k}-\mathcal{H}_{k}(\boldsymbol{x})\right),
\end{aligned}
$$

where $\boldsymbol{x}_{k}^{b}$ is a prior, or background, estimate of the state of the system (generally obtained by propagating forward in time the previous analysis $\boldsymbol{x}_{k-1}^{a}$ with Eq. 16). The error in the prior estimate is assumed to be unbiased with covariance matrix $\mathbf{B}_{k}$ and to be uncorrelated to errors in the observations.

We take the observation operator $\mathcal{H}_{k}$ to be linear around $\boldsymbol{x}_{k}^{b}$, meaning that

$\mathcal{H}_{k}(\boldsymbol{x}) \approx \mathcal{H}_{k}\left(\boldsymbol{x}_{k}^{b}\right)+\mathbf{H}_{k}\left(\boldsymbol{x}-\boldsymbol{x}_{k}^{b}\right)$,

where $\mathbf{H}_{k}$ is the linearisation of the observation operator about the background $\boldsymbol{x}_{k}^{b}$. Under this assumption, the cost function has an explicit minimum

$\boldsymbol{x}_{k}^{a}=\boldsymbol{x}_{k}^{b}+\mathbf{K}_{k}\left(\boldsymbol{y}_{k}-\mathcal{H}_{k}\left(\boldsymbol{x}_{k}^{b}\right)\right)$,

where

$\mathbf{K}_{k}=\mathbf{B}_{k} \mathbf{H}_{k}^{T}\left(\mathbf{H}_{k} \mathbf{B}_{k} \mathbf{H}_{k}^{T}+\mathbf{R}_{k}\right)^{-1}$.

The analysis error covariance matrix can be estimated as

$\mathbf{P}_{e, k}=\left(\mathbf{I}-\mathbf{K}_{k} \mathbf{H}_{k}\right) \mathbf{B}_{k}$.

In theory, the true background error covariance matrix $\mathbf{B}_{k}$ should be updated at each time step. However, this process is extremely expensive for real-time applications and, instead, we use a matrix with a simplified structure specified by the user. We will see in the numerical experiments (Sects. 4 and 5) how setting $\mathbf{B}_{k}$ appropriately is essential in order to obtain good estimates. Although the assimilation scheme we propose here to use with the moving mesh method is a variant of the traditional non-linear 3D-Var method, it is in essence a variational method with a fixed form for the background covariance matrices and we will refer to it as the 3D-Var method in the rest of the paper.

\subsubsection{Ensemble transform Kalman filter}

The ensemble Kalman filter (EnKF) introduced by Evensen (1994) approximates a fully non-linear Monte Carlo filter. At each time step, the state of the system is represented by an ensemble of $N_{e}$ realisations $\left\{\boldsymbol{x}_{k}^{(i)}, i=1, \ldots, N_{e}\right\}$. The state estimate is given by the ensemble mean

$\overline{\boldsymbol{x}}_{k}=\frac{1}{N_{e}} \sum_{i=1}^{N_{e}} \boldsymbol{x}_{k}^{(i)}$,

and the state error covariance matrix by the ensemble covariance matrix

$\mathbf{P}_{e, k}=\frac{1}{N_{e}-1} \mathbf{X}_{k} \mathbf{X}_{k}^{T}$,

where $\mathbf{X}_{k}$ is the anomalies matrix defined as

$\mathbf{X}_{k}=\left[\boldsymbol{x}_{k}^{(1)}-\overline{\boldsymbol{x}}_{k}, \ldots, \boldsymbol{x}_{k}^{\left(N_{e}\right)}-\overline{\boldsymbol{x}}_{k}\right]$.

From the ensemble covariance matrix, we can define the matrix Corr that contains an estimate of the correlation between the state variables to be

$[\text { Corr }]_{i, j}=\frac{\left[\mathbf{P}_{e, k}\right]_{i, j}}{\sqrt{\left[\mathbf{P}_{e, k}\right]_{i, i}\left[\mathbf{P}_{e, k}\right]_{j, j}}}$,

where $[\mathbf{C o r r}]_{i, j}$ and $\left[\mathbf{P}_{e, k}\right]_{i, j}$ denote the entry in the $i$ th row and $j$ th column of Corr and $\mathbf{P}_{e, k}$, respectively.

The forecast step propagates the ensemble from time $t_{k}$ to $t_{k+1}$ with the non-linear model $\mathcal{M}_{k, k+1}$. For the analysis step, we use the efficient ETKF introduced by Bishop et al. (2001) and follow the implementation of the algorithm given by Hunt et al. (2007).

The ETKF may generate ensembles of analyses with underestimated spread, which can lead to the divergence of the filter. We use an inflation procedure (Anderson and Anderson, 1999) here to avoid this potential degeneracy. In the rest of the paper, the inflation factor is denoted by the parameter $\lambda_{\text {infla. }}$.

In the twin experiments performed in Sects. 4 and 5, we use a large number of ensembles to avoid producing spurious correlations in $\mathbf{P}_{e, k}$. Therefore, no localisation has been employed in this paper.

\subsection{Form of the state vector in the moving mesh case}

Traditionally, in a data assimilation scheme, the state vector includes all the physical variables of the given dynamical system. For a fixed-grid numerical method, the model variables are defined at fixed spatial positions. For example, for a grounded ice sheet modelled with a fixed-grid method (and assuming every parameter is perfectly known), the unknown variables are the ice thicknesses located at known positions (see, e.g. Bonan et al., 2014). 
In contrast, the primary characteristic of a moving point method is that the numerical domain evolves in time. The positions of the nodes evolve jointly with the model variables (such as ice thickness) according to the dynamical system equations and can be updated using the assimilation scheme. We therefore include the positions of the nodes in the state vector. As a consequence, we define the state vector $\boldsymbol{x}$ as follows:

$$
\boldsymbol{x}=\left(\begin{array}{c}
\boldsymbol{x}_{h} \\
\boldsymbol{x}_{r}
\end{array}\right) \text { with } \boldsymbol{x}_{h}=\left(\begin{array}{c}
h_{1} \\
\vdots \\
h_{n_{r}-1}
\end{array}\right) \text { and } \boldsymbol{x}_{r}=\left(\begin{array}{c}
\hat{r}_{2} \\
\vdots \\
\hat{r}_{n_{r}}
\end{array}\right)
$$

Estimates obtained by combining DA with this formulation of $\boldsymbol{x}$ using a moving point numerical model provide more information on the state of the system than if we were using a fixed-grid method.

In particular, for an ice sheet model, this approach gives us a direct estimation of the position of the ice sheet margin that cannot be obtained in fixed-grid methods without interpolation. In this case, we do not include in $\boldsymbol{x}$ the ice thickness at the margin $h_{n_{r}}$ or the position of the ice divide $\hat{r}_{1}$ as both are fixed to zero. The DA schemes must, however, provide estimates with strictly positive ice thicknesses $h_{i}, i=1, \ldots, n_{r}-1$ and a preserved order for node positions to respect the assumption of the moving mesh scheme.

This can be achieved with the 3D-Var method if the specified background covariance matrix $\mathbf{B}_{k}$ in Eq. (21) is prescribed carefully. At time $t_{k}$, we decompose the background error covariance matrix $\mathbf{B}$ and the tangent linear matrix of the observation operator $\mathbf{H}$ (we drop the time index $k$ for clarity) as

$$
\begin{aligned}
& \mathbf{B}=\left(\begin{array}{cc}
\mathbf{B}_{h} & \mathbf{B}_{r h}^{T} \\
\mathbf{B}_{r h} & \mathbf{B}_{r}
\end{array}\right) \text { and } \mathbf{H}=\left(\begin{array}{cc}
\mathbf{H}_{h} & \mathbf{H}_{r}
\end{array}\right)= \\
& \left(\frac{\partial \mathcal{H}}{\partial \boldsymbol{x}_{h}}\left(\boldsymbol{x}^{f}\right) \frac{\partial \mathcal{H}}{\partial \boldsymbol{x}_{r}}\left(\boldsymbol{x}^{f}\right)\right),
\end{aligned}
$$

where $\mathbf{B}_{h}$ is the background error covariance matrix between the model variables, $\mathbf{B}_{r}$ is the error covariance between mesh point locations and $\mathbf{B}_{r h}$ includes the cross-covariances between errors in point locations and errors in model variables. The different components of the state vector are then updated by the following analysis step:

$$
\begin{aligned}
\boldsymbol{x}_{h}^{a}=\boldsymbol{x}_{h}^{b}+\left(\mathbf{B}_{h} \mathbf{H}_{h}^{T}+\mathbf{B}_{r h}^{T} \mathbf{H}_{r}^{T}\right)\left(\mathbf{H B H} \mathbf{H}^{T}+\mathbf{R}\right)^{-1} \\
\quad\left(\boldsymbol{y}-\mathcal{H}\left(\boldsymbol{x}^{b}\right)\right) \\
\boldsymbol{x}_{r}^{a}=\boldsymbol{x}_{r}^{b}+\left(\mathbf{B}_{r h} \mathbf{H}_{h}^{T}+\mathbf{B}_{r} \mathbf{H}_{r}^{T}\right)\left(\mathbf{H B H}^{T}+\mathbf{R}\right)^{-1} \\
\quad\left(\boldsymbol{y}-\mathcal{H}\left(\boldsymbol{x}^{b}\right)\right) .
\end{aligned}
$$

The most difficult step with this form of analysis is, in general, to set appropriately the cross-covariances in $\mathbf{B}_{r h}$ that are needed for the update stage. For example, if either $\mathbf{H}_{h}$ or $\mathbf{H}_{r}$ is zero, a non-zero $\mathbf{B}_{r h}$ matrix is the only way to correct estimates of both $\boldsymbol{x}_{h}$ and $\boldsymbol{x}_{r}$. However, we will see in the next section that in our assimilation systems for the ice sheet model, the observation operator depends explicitly on both ice thickness variables and mesh node locations, and therefore by setting $\mathbf{B}_{r h}$ to zero we can still obtain good estimates.

For the moving point ice sheet model, the DA analysis step updates both ice thickness variables and node positions, but the total mass and mass fractions have to be updated as well, since they are not preserved by the analysis (and there is no reason to preserve them). Therefore, these quantities need to be "reset" from the analysed state vector. This is easily done by using Eqs. (14) and (15). The adapted 3D-Var scheme is performed according to the following steps:

1. calculate a forecast of the state vector $\boldsymbol{x}^{b}$ by using the previous analysis solution to initialise the numerical moving point model,

2. use the analysis scheme (Eqs. 29 and 30) to produce the analysis $\boldsymbol{x}^{a}$,

3. from $\boldsymbol{x}^{a}$, calculate the analysed total mass $\theta^{a}$ and update the mass fractions $\mu^{a}$ using Eqs. (14) and (15),

4. evolve the analysis solution using the numerical moving point model to the next time where observations are available and

\section{5. repeat steps $2-5$.}

The adapted ETKF roughly follows the same path as 3DVar except that, at step 1, we calculate the forecast for each member of the ensemble and, at step 3, the total mass and mass fractions have to be updated for each member of the ensemble (they are different for each ensemble member). The background error covariance is also updated using the ensemble statistics. The strict positivity of ice thickness variables and the order required in Eq. (13) for node positions are ensured by appropriately setting the initial ensemble in the ETKF.

We remark that observations outside the domain of the background state at the time of the update cannot be assimilated. This is a limitation on both methods, but the ETKF has the advantage that it can take into account such observations if the domain of the background of any member of the ensemble is large enough to include the reference domain.

\subsection{Type of observations assimilated}

In the twin experiments performed in Sects. 4 and 5, we use three different conventional types of observations of an ice sheet system that are available in reality (see, e.g. Vaughan et al., 2013). The first is direct observations of the ice thickness. Assuming that we have an observation of the ice thickness located at position $r^{o}$, we define the associated observa- 
tion operator as

$\mathcal{H}(\boldsymbol{x})= \begin{cases}h_{i}+\frac{r^{o}-\hat{r}_{i}}{\hat{r}_{i+1}-\hat{r}_{i}}\left(h_{i+1}-h_{i}\right) & \text { if } \hat{r}_{i} \leq r^{o} \leq \hat{r}_{i+1} \\ 0 & \text { elsewhere }\end{cases}$

which is merely a piecewise linear interpolation operator. Note that $\mathcal{H}$ depends on both ice thickness variables $h_{i}$ and node locations $\hat{r}_{i}$. We also assimilate observations of surface elevation and surface ice velocity. We again use a piecewise linear interpolation operator as in Eq. (31). For observations of surface elevation, we have

$\mathcal{H}(\boldsymbol{x})= \begin{cases}s_{i}+\frac{r^{o}-\hat{r}_{i}}{\hat{r}_{i+1}-\hat{r}_{i}}\left(s_{i+1}-s_{i}\right) & \text { if } \hat{r}_{i} \leq r^{o} \leq \hat{r}_{i+1} \\ b\left(r_{i}\right) & \text { elsewhere }\end{cases}$

with

$s_{i}=h_{i}+b\left(r_{i}\right)$.

For observations of surface ice velocity, from a discretisation of Eq. (4) (see Appendix B2 in Bonan et al., 2016), we have

$\mathcal{H}(\boldsymbol{x})= \begin{cases}u_{s, i}+\frac{r^{o}-\hat{r}_{i}}{\hat{r}_{i+1}-\hat{r}_{i}}\left(u_{s, i+1}-u_{s, i}\right) & \text { if } \hat{r}_{i} \leq r^{o} \leq \hat{r}_{i+1} \\ 0 & \text { elsewhere }\end{cases}$

with

$$
\begin{aligned}
u_{s, i} & =\frac{1}{2} A\left(\rho_{\mathrm{i}} g\right)^{3} \operatorname{sgn}\left(s_{n_{r}}-s_{n_{r}-1}\right) \\
& h_{i}^{4}\left(\frac{\partial b}{\partial r}\left(r_{i}\right)\right)^{3}+\frac{3}{5} \frac{h_{i}^{5}-h_{i-1}^{5}}{\hat{r}_{i}-\hat{r}_{i-1}}\left(\frac{\partial b}{\partial r}\left(r_{i}\right)\right)^{2} \\
& +\frac{1}{3}\left(\frac{h_{i}^{3}-h_{i-1}^{3}}{\hat{r}_{i}-\hat{r}_{i-1}}\right)^{2} \frac{\partial b}{\partial r}\left(r_{i}\right)+\frac{27}{343}\left(\frac{h_{i}^{7 / 3}-h_{i-1}^{7 / 3}}{\hat{r}_{i}-\hat{r}_{i-1}}\right)^{3} \mid,
\end{aligned}
$$

except for $u_{s, 1}=0$.

We may also assimilate observations of the position of the ice sheet margin. Using a moving point method allows the movement of boundaries to be tracked explicitly. In our context, the position of the ice sheet margin is represented by $\hat{r}_{n_{r}}$. As a consequence, the observation operator for such an observation is defined by

$\mathcal{H}(\boldsymbol{x})=\hat{r}_{n_{r}}$.

The operator is continuous and linear. This makes the assimilation of the position of the margin straightforward in comparison with the same assimilation with a fixed-grid model (see, e.g. Lecavalier et al., 2014).

\section{Numerical experiments with an idealised model}

To demonstrate the efficiency of our DA approach, we perform twin experiments with two different configurations. In this section, we consider experiments using an idealised system with a flat bedrock and the EISMINT surface mass balance detailed in Eq. (A1).

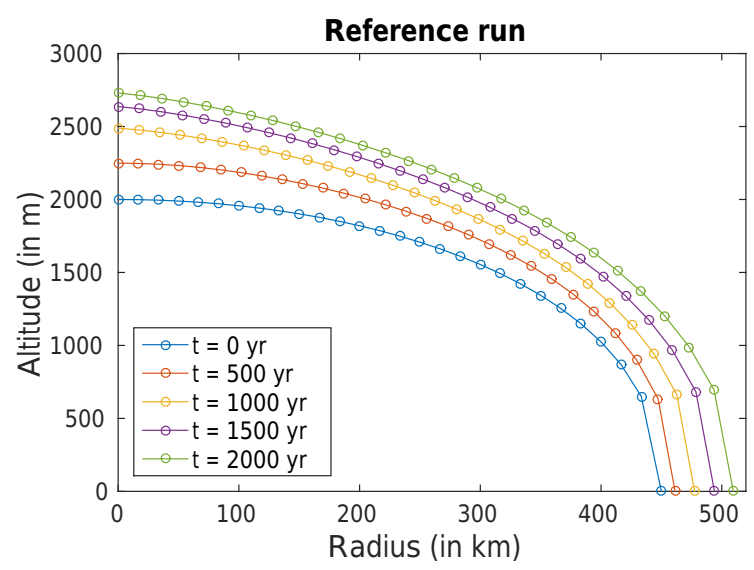

Figure 2. Ice thickness profile from the reference run in a simple case (flat bedrock, EISMINT surface mass balance from Eq. A1). The initial state follows the profile of Eq. (37) with $h_{0}=2000 \mathrm{~m}$ and $r_{\max }=450 \mathrm{~km}$. The reference run is obtained with an initial mesh of $n_{r}=28$ points evenly spaced between $\hat{r}_{1}=0$ and $\hat{r}_{n_{r}}=$ $450 \mathrm{~km}$.

\subsection{Experimental design}

We first generate a model run with the moving point numerical model from known initial conditions. From this simulation, observations are created with added error sampled from a Gaussian distribution. This run is used as a reference to measure the quality of the DA estimates.

We define the reference initial ice thickness profile by the function

$h(0, r)=h_{0}\left(1-\left(\frac{r}{r_{\max }}\right)^{2}\right)^{3 / 7} \quad 0 \leq r \leq r_{\max }$

where $h_{0}=2000 \mathrm{~m}$ and $r_{\max }=450 \mathrm{~km}$. This function gives a smooth interior profile with a steep snout at the ice sheet margin $r_{\max }$. This is in compliance with the physics involved in the ice sheet model and provides an initial state with a margin that is immediately in motion. The reference run is obtained with an initial mesh of $n_{r}=28$ points evenly spaced between $\hat{r}_{1}=0$ and $\hat{r}_{n_{r}}=450 \mathrm{~km}$. The model time step is $\Delta t=0.02$ years, the bed elevation $b$ is fixed to zero and the surface mass balance used is from the EISMINT benchmark (Eq. A1). The experiment starts at time $t=0$ years and ends at $t=2000$ years. The evolution of the reference ice thickness profile can be seen in Fig. 2.

From the reference run, we generate observations of ice thickness and the position of the ice sheet margin at times $t_{1}=500$ and $t_{2}=1500$ years. Observations of thickness are taken at each point except at the margin (a total of $27 \mathrm{ob}$ servations) with added random noise from the Gaussian distribution $\mathcal{N}\left(0, \sigma_{h}^{o 2}\right), \sigma_{h}^{o}=100 \mathrm{~m}$. For the position of the margin, the observational noise is sampled from $\mathcal{N}\left(0, \sigma_{r}^{o 2}\right)$, $\sigma_{r}^{o}=10 \mathrm{~km}$. 
To evaluate the performance of our DA approaches, we compare the estimated ice thickness profiles with their reference counterparts. This is mostly done graphically. We also study the quality of the estimates of two variables: the ice thickness at the ice divide at $r=0$ and the position of the ice sheet margin.

\subsection{Updating the ice thickness only}

We begin by studying the performance of the DA schemes in the idealised configuration where we assimilate observations of ice thickness only. We start with an experiment using the 3D-Var algorithm in which only the ice thickness is updated at the assimilation times and the mesh point positions are not updated.

The background state is defined as follows:

- At initial time, the background ice thickness profile is set using the same profile as the reference (Eq. 37) but with $h_{0}=2100 \mathrm{~m}(+5 \%$ error from the reference $)$ and $r_{\max }=472.5 \mathrm{~km}$ (also $+5 \%$ error $)$.

- The background mesh consists of $n_{r}=28$ points, evenly spaced between $\hat{r}_{1}=0$ and $\hat{r}_{n_{r}}=472.5 \mathrm{~km}$ at initial time.

- The model time step is $\Delta t=0.02$ years.

As we are using a 3D-Var scheme in this experiment, the background error covariance matrix $\mathbf{B}$ needs to be prescribed at both times of assimilation $\left(t_{1}=500\right.$ and $t_{2}=1500$ years $)$. In this first experiment, we only update ice thickness variables, so we set the background error covariance matrix for point positions $\mathbf{B}_{r}$ and the cross-covariance matrix $\mathbf{B}_{r h}$ to zero. We define $\mathbf{B}_{h}$ the covariance matrix for ice thickness variables as

$\mathbf{B}_{h}=\mathbf{D}_{h}^{1 / 2} \mathbf{C}_{h} \mathbf{D}_{h}^{1 / 2}$,

with $\mathbf{D}_{h}$ the diagonal variance matrix and $\mathbf{C}_{h}$ the correlation matrix. $\mathbf{D}_{h}$ is simply set to $\sigma_{h}^{b^{2}} \mathbf{I}_{n_{r}-1}$ with $\sigma_{h}^{b}=100 \mathrm{~m}$. The background error correlation structure follows a secondorder autoregressive (SOAR) distribution with

$$
\begin{gathered}
{\left[\mathbf{C}_{h}\right]_{i, j}=\left(1+\frac{\left|\hat{r}_{i}^{b}-\hat{r}_{j}^{b}\right|}{L_{h}}\right) \exp \left(-\frac{\left|\hat{r}_{i}^{b}-\hat{r}_{j}^{b}\right|}{L_{h}}\right)} \\
i, j=1, \ldots, n_{r}-1,
\end{gathered}
$$

where $\left[\mathbf{C}_{h}\right]_{i, j}$ denotes the entry in the $i$ th row and $j$ th column of $\mathbf{C}_{h}, \hat{r}_{i}^{b}$ the location of the $i$ th mesh point of the background state at the time of assimilation and $L_{h}$ is some correlation length scale to be fixed. The SOAR function is preferred to a Gaussian structure as the matrix $\mathbf{C}_{h}$ is better conditioned for inversion in that case (Haben et al., 2011). We set $L_{h}$ to $100 \mathrm{~km}$.

This definition of $\mathbf{B}$ takes into account the flow dependency of the moving point locations, making our approach adaptive. Figure 3 displays $\mathbf{B}_{h}$ at assimilation times $t_{1}=500$ and $t_{2}=1500$ years. As the distance between grid points increases in time in the experiment, the covariances tend to reduce between the two assimilation times. For example, the covariance between the location of points $\hat{r}_{1}^{b}$ and $\hat{r}_{n_{r}-1}^{b}$ is reduced from $\left[\mathbf{B}_{h}\right]_{1, n_{r}-1}=530.7$ at $t_{1}=500$ years to $\left[\mathbf{B}_{h}\right]_{1, n_{r}-1}=446.6$ at $t_{2}=1500$ years. In addition, we note decreased correlations for points around the centre of the mesh due to a greater distance between adjacent nodes in the centre of the grid than at the boundaries.

The formulation of $\mathbf{B}$ forces the recomputation of the matrix at every assimilation time. This is a limiting factor of our 3D-Var approach, especially for high-dimensional systems, making it cost more than traditional 3D-Var for fixed-grid models in which B is only computed once. Nevertheless, our experiments demonstrate that this formulation of the background error covariance matrix ensures that the moving point framework produces positive estimates of ice thickness variables and a smooth interior profile in accordance with the physics of the system.

We now evaluate the quality of the estimates. Figure 4 (left) displays the analysed ice thickness profile compared to its background and reference counterparts at the first time of assimilation $t_{1}=500$ years. The picture shows that the ice thickness profile in the interior of the ice sheet is substantially improved by DA. For example, the absolute error in ice thickness at the ice divide $(r=0)$ is reduced from 100 to $58.3 \mathrm{~m}$ by the $3 \mathrm{D}$-Var analysis. Results are even better between $r=100$ and $400 \mathrm{~km}$. Since we only update $\boldsymbol{x}_{h}$ in this experiment, the position of the margin is not modified by our update. Nevertheless, by correcting the interior of the ice sheet, the forecast of the migration of the margin is improved (see the central and right pictures after $t=500$ years; Fig. 4), and at the second assimilation time, $t=1500$ years, the absolute difference between the position of the margin before analysis and its reference position is only $5.6 \mathrm{~km}$ (instead of $15.9 \mathrm{~km}$ without DA)

\subsection{Updating ice thickness variables and node positions}

We now use 3D-Var to update both ice thickness variables and node locations. The definitions of $\mathbf{B}_{h}$ and $\mathbf{B}_{r h}$ remain the same as in the previous experiment, but we set the covariance matrix for node positions $\mathbf{B}_{r}$ to be $\mathbf{B}_{r}=\mathbf{D}_{r}^{1 / 2} \mathbf{C}_{r} \mathbf{D}_{r}^{1 / 2}$ with $\mathbf{D}_{r}$ the diagonal variance matrix and $\mathbf{C}_{r}$ a correlation matrix. The matrix $\mathbf{D}_{r}$ is set to $\sigma_{r}^{b^{2}} \mathbf{I}_{n_{r}-1}$ with $\sigma_{r}^{b}=22.5 \mathrm{~km}$ and $\mathbf{C}_{r}$ follows a SOAR distribution with

$$
\begin{gathered}
{\left[\mathbf{C}_{r}\right]_{i, j}=\left(1+\frac{\left|\hat{r}_{i+1}^{b}-\hat{r}_{j+1}^{b}\right|}{L_{r}}\right) \exp \left(-\frac{\left|\hat{r}_{i+1}^{b}-\hat{r}_{j+1}^{b}\right|}{L_{r}}\right),} \\
i, j=1, \ldots, n_{r}-1,
\end{gathered}
$$

where $L_{r}$ is a correlation length scale fixed to $100 \mathrm{~km}$. The correlation matrix $\mathbf{B}_{r}$ constrains the movement of the assimilated mesh points and the correlation function used in the 

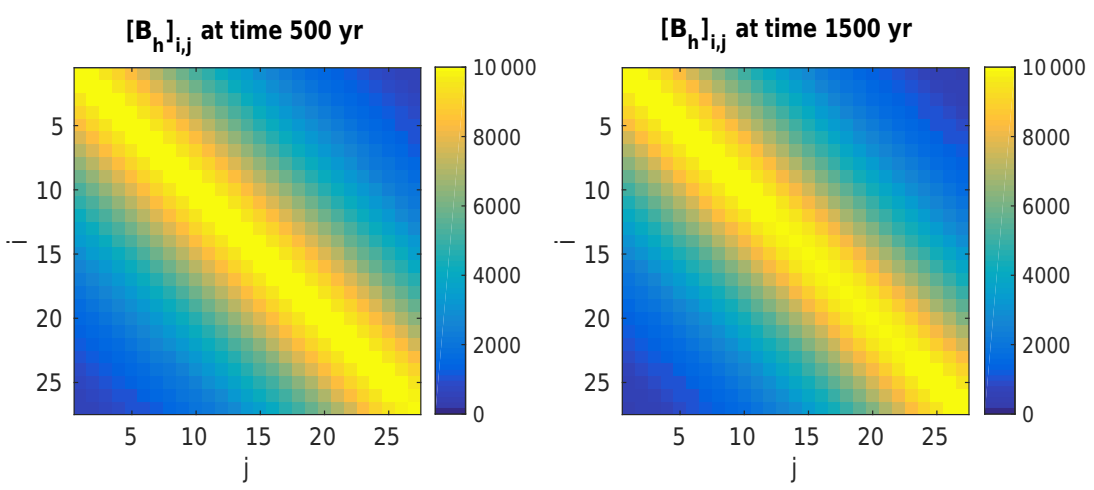

Figure 3. Covariance matrices for ice thickness variables $\mathbf{B}_{h}$ used by the 3D-Var at assimilation times $t_{1}=500$ and $t_{2}=1500$ years. Covariances between variables at distant locations tend to reduce between the two assimilation times. The distance between adjacent nodes also tends to be greater in the centre of the mesh than at the boundaries, leading to a decreasing covariance at $t_{2}=1500$ years in this area.

(a) 3D-Var update, $\mathrm{t}=500 \mathrm{yr}$

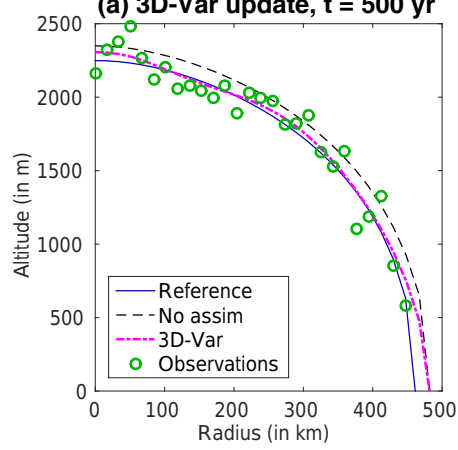

(b) Evolution position margin

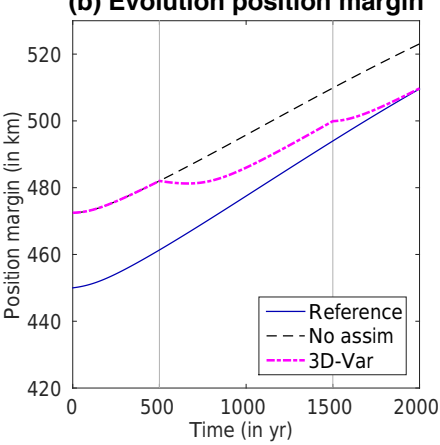

(c) Evolution grid with 3D-Var

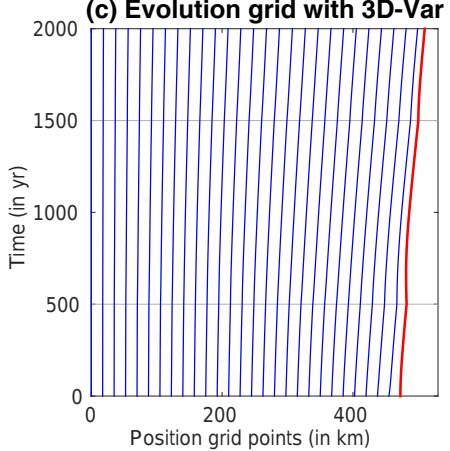

Figure 4. (a) 3D-Var analysis at time $t=500$ years compared with the forecast and the reference when we update only ice thickness variables. The ice thickness profile is improved, especially between $r=100$ and $r=400 \mathrm{~km}$. (b) Evolution of the position of the margin with time. Even if the position of the margin is not directly updated, the trajectory of the margin is corrected as a result of the ice thickness update. (c) Evolution of the position of grid points with time. The trajectory of each grid node is corrected after each analysis, as is the margin.

formulation of $\mathbf{B}_{r}$ is selected to ensure that the order of the points defined by Eq. (13) is preserved by the 3D-Var algorithm. Since the distance between nodes evolves in time, it is even more important than in the previous case to use a flowdependent background error covariance matrix $\mathbf{B}$.

Results for the ice thickness profile are shown in Fig. 5. Overall estimates obtained with updating both ice thickness variables and node positions are better than when we update only ice thickness variables. The absolute error in ice thickness at the ice divide $(r=0)$ is reduced from 100 to $60.2 \mathrm{~m}$ by the $3 \mathrm{D}$-Var analysis at time $t_{1}=500$ years, which is similar to the previous experiment. However, we now obtain at $t_{1}=500$ years a very accurate ice thickness profile close to the margin and its estimated position has an absolute error of only $0.2 \mathrm{~km}$. This shows that the estimated position of the ice sheet margin can be accurately corrected by only using standard observations (no observation of the position of the margin is involved in this experiment). At the second time of assimilation at $t_{2}=1500$ years, the estimate is degraded, however, as a result of using fixed variances in the matrix $\mathbf{B}$. This behaviour is discussed further in Sect. 4.5.
The 3D-Var method provides information on the analysis covariance structures for ice thickness variables and mesh point positions. In Fig. 6, we display the estimated standard deviations and the error correlation matrix Corr (see Eq. 26) obtained at time $t=500$ years using the estimated analysis error covariance matrix $\mathbf{P}_{e, k}$ given by Eq. (22). We see that the 3D-Var method produces decreased standard deviations and correlation length scales for ice thickness variables close to the ice divide and decreased standard deviations and correlation length scales for node locations close to the margin. The 3D-Var method also produces strong anti-correlations between ice thickness variables and node positions, meaning that in order to fit the observations where the ice thickness variables become larger, the associated nodes need to retreat.

In these experiments, we have specified a fixed form for the background error covariance matrices, which are defined in terms of the positions of the nodes. We next show, using an ETKF, how the covariances are expected to evolve in time with the model dynamics and the effects of this on the assimilation. 
(a) 3D-Var update, $\mathrm{t}=\mathbf{5 0 0} \mathrm{yr}$

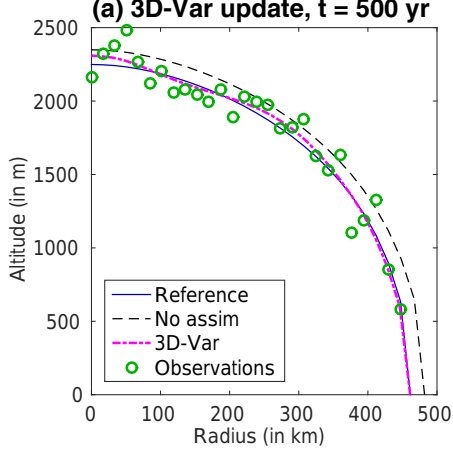

(b) Evolution position margin

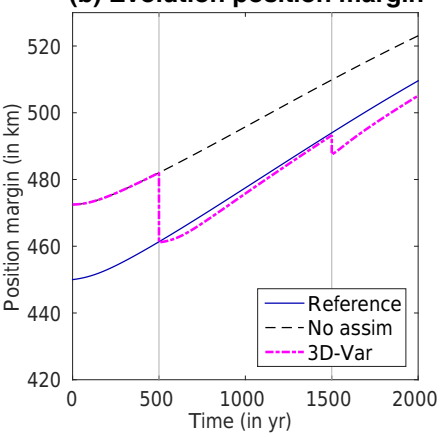

(c) Evolution grid with 3D-Var

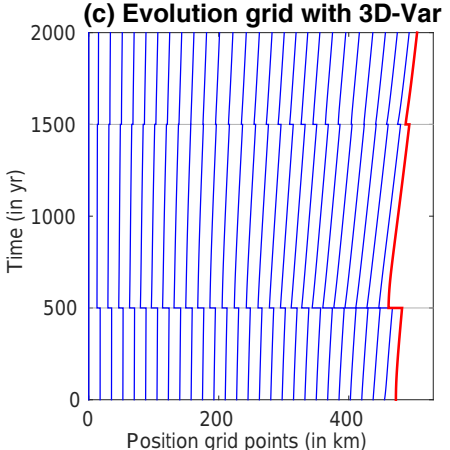

Figure 5. (a) 3D-Var analysis at time $t=500$ years compared with the forecast and the reference when we update ice thickness variables and node locations. In contrast to the results shown in Fig. 4, the ice thickness profile is substantially improved close to the margin. (b) Evolution of the position of the margin with time. The estimates are of very good quality even if the margin is not observed directly. (c) Evolution of the position of mesh points with time. The trajectory of each node is corrected by each analysis, as is the margin.
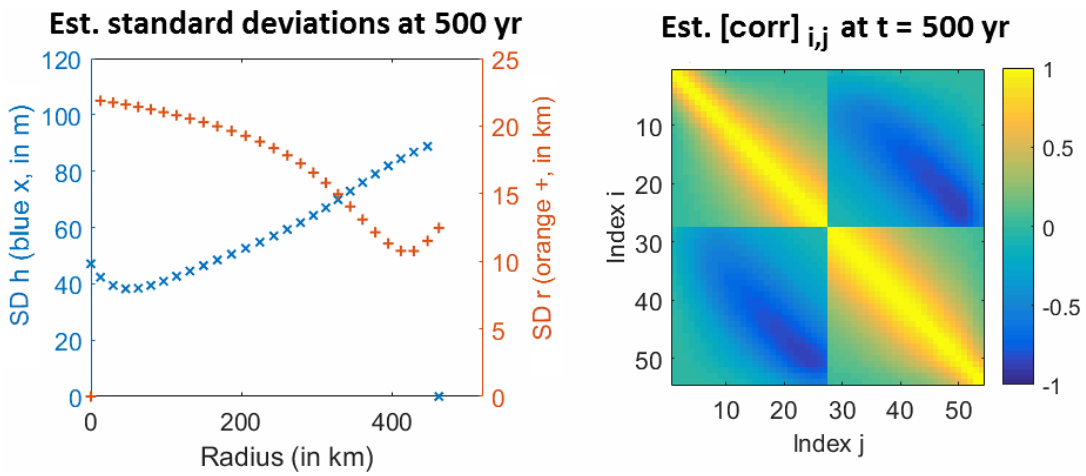

Figure 6. Standard deviations and correlation matrix Corr estimated from the 3D-Var analysis at time $t=500$ years when we use only observations of ice thickness. Auto-correlations between ice thicknesses are located in the top left corner of Corr; auto-correlations between node positions are in the bottom right corner. The rest of the matrix depicts the cross-correlations.

\subsection{Using an ETKF}

We now perform the same experiment as before except that we now use an ETKF. The key question is how to generate the initial ensemble composed of $N_{e}$ members. The easiest way is to add noise to a background state sampled from a Gaussian law $\mathcal{N}(\boldsymbol{0}, \mathbf{B})$ with $\mathbf{B}$ as the background error covariance matrix defined in Eq. (28).

In this experiment, we generate an initial ensemble of $N_{e}=200$ members using:

- the same background state used in the experiments detailed in Sect. 4.2 and 4.3,

- $\mathbf{B}_{h}$ defined by Eq. (38) with the diagonal matrix $\mathbf{D}_{h}=$ $\sigma_{h}^{2} \mathbf{I}_{n_{r}-1}, \sigma_{h}^{b}=100 \mathrm{~m}, \mathbf{C}_{h}$ defined by Eq. (39), $L_{h}=$ $100 \mathrm{~km}$,
- $\mathbf{B}_{r}$ taken as $\mathbf{D}_{r}^{1 / 2} \mathbf{C}_{r} \mathbf{D}_{r}^{1 / 2}$ with $\mathbf{C}_{r}$ defined by Eq. (40) with $L_{h}=100 \mathrm{~km}$ and the diagonal matrix $\mathbf{D}_{r}$ defined as

$$
\left[\mathbf{D}_{r}\right]_{i i}=\min \left(\sigma_{r}^{b}, \alpha \hat{r}_{i}\right) \quad i=1, \ldots, n_{r}-1
$$

with $\sigma_{r}^{b}=22.5 \mathrm{~km}$ and $\alpha=0.2$, and

$-\mathbf{B}_{r h}$ set to zero.

Note that the definition of $\mathbf{B}$ is slightly different from the previous experiment as we choose different diagonal variances. The change is because of the high probability of generating useless initial meshes with negative radii using $\mathbf{D}_{r}=\sigma_{r}^{b^{2}} \mathbf{I}_{n_{r}-1}$, as the background standard deviation $\sigma_{r}^{b}$ is larger than the background position of the first points (for example, $\hat{r}_{2}^{b}=17.5 \mathrm{~km}$ ). To avoid this problem, we have decided just to reduce the variance for the position of points near the ice divide using Eq. (41). The new ensemble mean has, at the initial time, an estimated position of the margin 

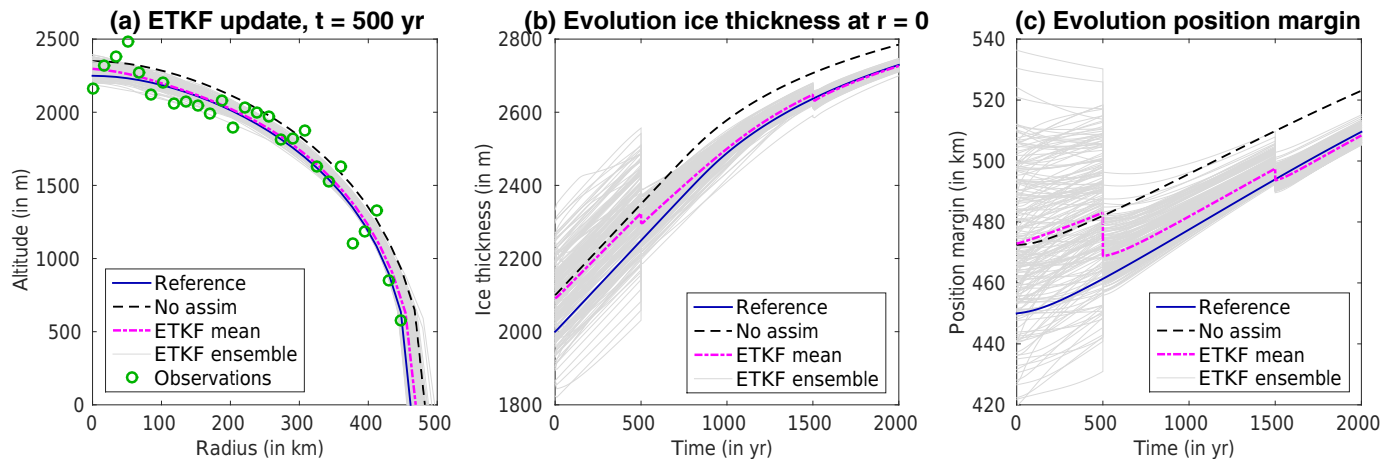

Figure 7. (a) ETKF analysis at time $t=500$ years compared with the forecast and the reference. The ice thickness profile is improved over the whole domain and the reference profile is within the ensemble spread. (b) Evolution of the ice thickness at $r=0$ with time. The estimates are of very good quality and the estimates seem to converge towards the reference value at the end of the study. (c) Evolution of the position of the margin with time. The ETKF provides consistent estimates and the reference value is always within the ensemble spread.
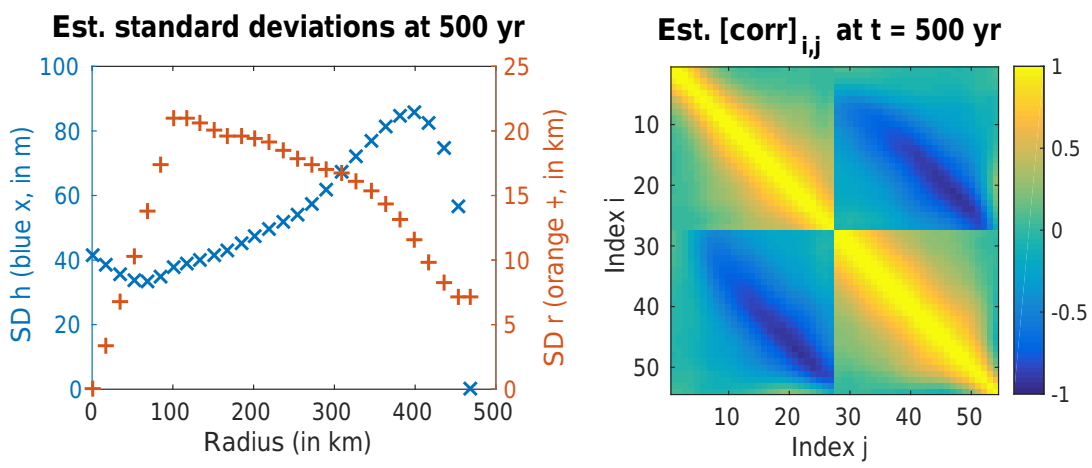

Figure 8. Standard deviations and correlation matrix Corr estimated from the ETKF analysis ensemble at time $t=500$ years when we use only observations of ice thickness. Auto-correlations between ice thicknesses are located in the top left corner of Corr; auto-correlations between node positions are in the bottom right corner. The rest of the matrix depicts the cross-correlations.

of $472.9 \mathrm{~km}$ with an estimated standard deviation of $22.8 \mathrm{~km}$ (where the true value at $t=0$ is $450 \mathrm{~km}$ ).

We do not use any inflation in this experiment $\left(\lambda_{\text {infla }}=1\right)$.

Results are summarised in Fig. 7. At the first time of assimilation $t_{1}=500$ years, the analysis step corrects the ice thickness profile well. The estimate of the ice thickness at $r=0$ is of the same quality as in the previous experiments (absolute error of $46.9 \mathrm{~m}$ ) and the estimate of the position of the margin is reduced from $483.1 \mathrm{~km}$ (forecast mean with estimated standard deviation $18.9 \mathrm{~km}$ ) to $468.8 \mathrm{~km}$ (analysis mean with estimated standard deviation $7.1 \mathrm{~km}$ ). The estimate obtained by the ETKF is in accordance with the true value (which is within the ensemble spread) and the absolute error of $7.5 \mathrm{~km}$ is of the same order as the estimated standard deviation. The rest of the experiment exhibits the same quality in terms of recovering the ice thickness profile.

The ETKF provides information on the covariance structures for ice thickness variables and mesh point positions. We display estimated standard deviations and an estimate of the correlation matrix Corr (see Eq. 26) in Fig. 8 for the analysis ensemble at time $t=500$ years. The ETKF produces de- creased standard deviations and correlation length scales for ice thickness variables close to the ice divide. For example, the standard deviation of the ice thickness at the ice divide is more than halved by the analysis, from $97.4 \mathrm{~m}$ before analysis to $41.6 \mathrm{~m}$. Decreased standard deviations and correlation length scales are also obtained for node locations but close to the margin in this case. The standard deviation for the position of the margin is reduced from $18.9 \mathrm{~km}$ to $7.1 \mathrm{~km}$ by the analysis. The ETKF also produces strong anti-correlations between ice thickness variables and node positions, meaning that where ice thickness variables become larger associated nodes need to retreat to fit the observations of ice thickness.

\subsection{Comparing 3D-Var and the ETKF}

We now compare the results from applying the 3D-Var and ETKF assimilation schemes in the case where we observe only the ice thickness. We focus on the accuracy of the estimated ice thickness at $r=0$ and the position of the margin.

Figure 9 shows the evolution of the absolute errors in the estimates of the ice thickness at $r=0$ and in the position of the margin for the ETKF and for 3D-Var, with and without 

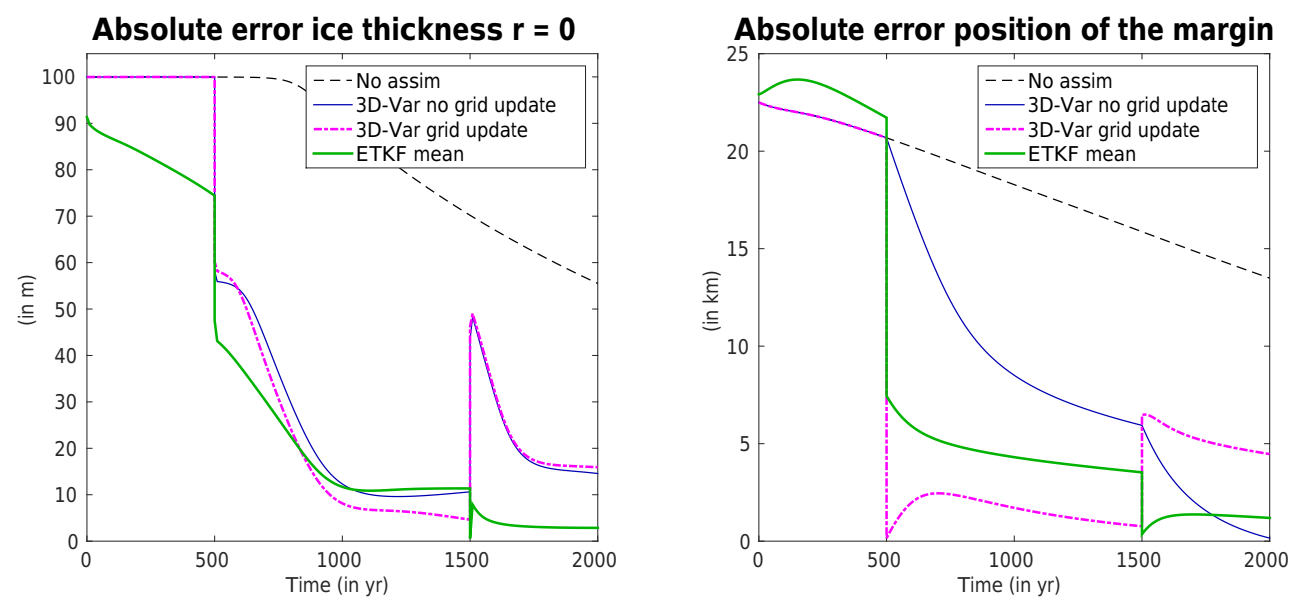

Figure 9. Evolution of the absolute error of the estimated ice thickness at $r=0$ and the estimated position of the margin when we observe only the ice thickness. We compare the absolute errors obtained when we use 3D-Var without and with correction of the position of grid nodes and when we use an ETKF.
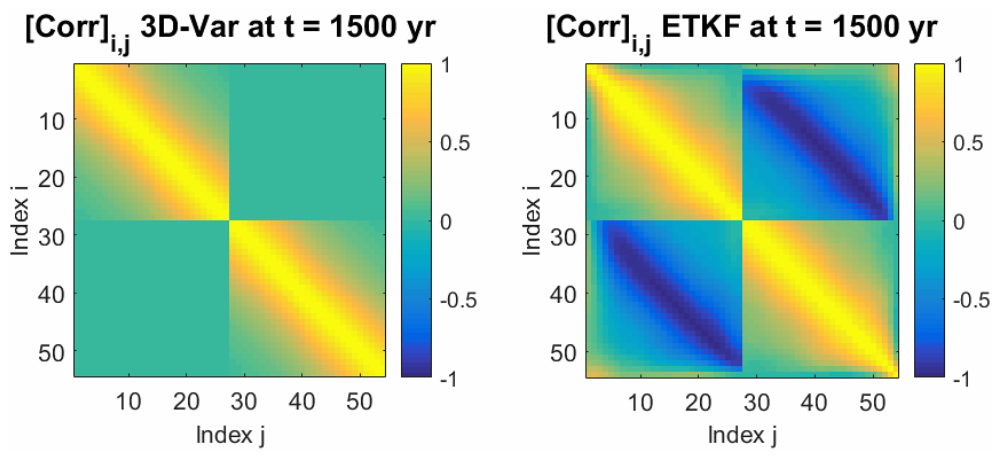

Figure 10. The background error covariance matrices used by the 3D-Var and ETKF methods to produce the analysis at time $t=1500$ years.

node updates. All three methods provide improved estimates at the first analysis time $\left(t_{1}=500\right.$ years $)$, leading to good forecasts up to the next assimilation time. We find that the ETKF tends to perform better than the variational approach and that for 3D-Var the estimates obtained by updating both ice thickness variables and node positions are generally better than those where only ice thickness variables are updated.

For 3D-Var without node updates, the analysis at the second time of assimilation $\left(t_{2}=1500\right.$ years $)$ of the ice thickness at $r=0$ is unfortunately degraded relative to the forecast, but the estimated position of the margin is still improved by the second analysis. In the case where ice thickness and nodes are updated, the estimates of both ice thickness at $r=0$ and the position of the margin are degraded at the second time of assimilation. This weakens the confidence in the forecast and we partially lose what we had gained from the previous analysis. The experiment shows the sensitivity of 3D-Var to current observations resulting from the dependence of the prescribed covariance matrix $\mathbf{B}$ on the positions of the mesh nodes.
Using the ETKF assimilation scheme, where the covariance matrix fully evolves in time, is seen to improve the overall estimates. At each assimilation time, the errors in the estimated ice thickness and the position of the margin are decreased. Notably, we do not observe any degrading of the estimates at the second time of assimilation. This improvement can be attributed to the better background forecast produced by the ETKF at each assimilation time.

In Fig. 10, we display the background error covariance matrices used by the 3D-Var and ETKF methods to produce the analysis at time $t=1500$ years. At the previous assimilation time $t=500$ years, the analysis covariances produced by both methods are very similar, as seen in Figs. 6 and 8 . However, because the 3D-Var error covariance matrix has a fixed form, the background covariance matrix used by 3DVar at the assimilation time $t=1500$ years has not changed significantly. In contrast, it can be seen that the ETKF background error covariance has fully evolved and contains much more information than the 3D-Var error covariance matrix. This explains the better ability of the ETKF to provide accurate estimates in the context of the moving point model. 

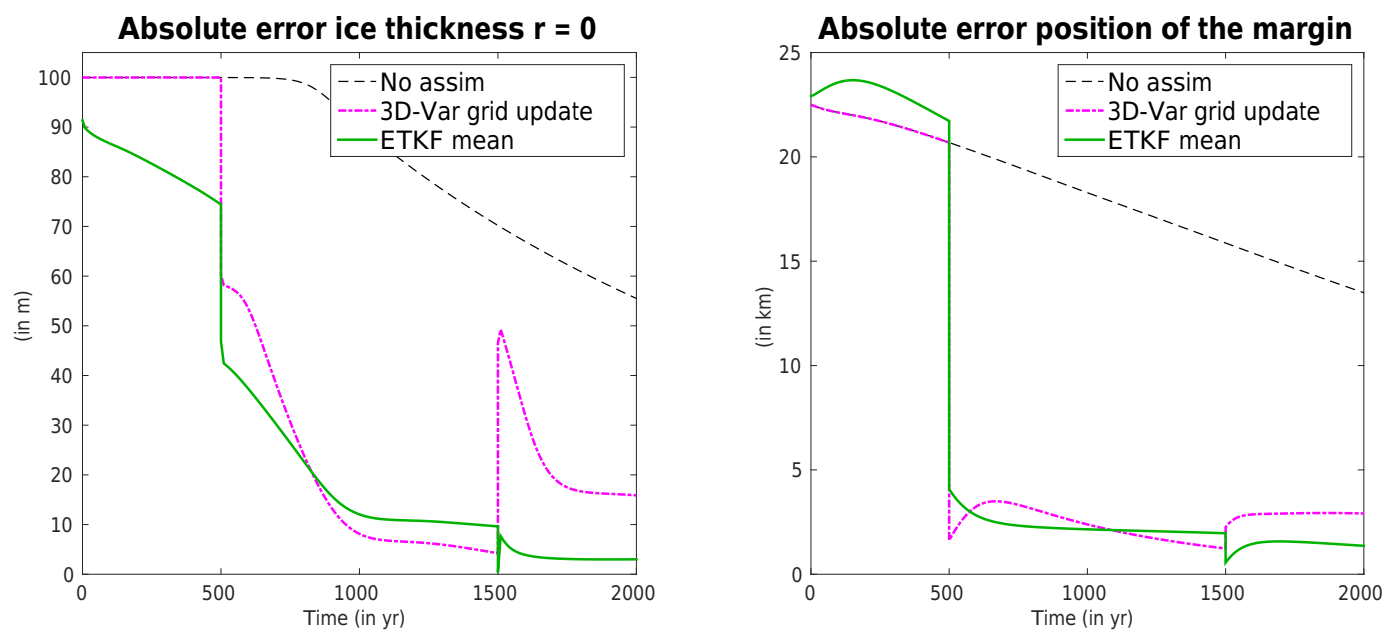

Figure 11. Evolution of the absolute error of the estimated ice thickness at $r=0$ and the estimated position of the margin when we observe the ice thickness and the position of the margin. We compare the absolute errors obtained when we use 3D-Var with correction of the position of grid nodes and when we use an ETKF. In both experiments, the results are improved with respect to the position of the margin (compared to results detailed in Fig. 9). No improvement (nor degradation) is observed for the ice thickness at $r=0$.

Propagating the background error covariances using the ensemble statistics ensures that the ETKF is a more reliable scheme than 3D-Var. This improvement has a computational cost, however, as we now need to run the model $N_{e}$ times instead of once for 3D-Var.

\subsection{Assimilating observations of the position of the margin}

In this section, we perform the same experiments as previously, but we now assimilate not only the same observations of ice thickness as before but also observations of the position of the margin. We consider only the case of 3D-Var with grid update and the ETKF.

Absolute errors for the estimates of the ice thickness at $r=0$ and the position of the margin are shown in Fig. 11 . In both cases, assimilating observations of the position of the margin is beneficial to our estimates of the margin and of the ice thickness profile close to the margin. For example, the estimated position of the margin at time $t=500$ years has an absolute error of $4.2 \mathrm{~km}$ for the ETKF (compared to $7.5 \mathrm{~km}$ previously). Not surprisingly, it does not change the results for the ice thickness at $r=0$.

Adding observations of the position of the margin in the data assimilation system reduces the estimated standard deviations obtained with the ETKF for variables close to the margin. For example, the estimated standard deviation for the position of the margin is now $5.8 \mathrm{~km}$ instead of $7.1 \mathrm{~km}$. Not surprisingly it has no influence on the standard deviation for variables close to the ice divide. The estimated correlation structure (not shown) is also not modified by adding observations of the position of the margin in the DA system.

\section{Numerical experiments with an advanced configuration}

In this section, we consider experiments using a more realistic configuration with a non-flat bedrock and an advanced surface mass balance, detailed in Appendix A2. We investigate the case of a rapidly warming climate over a short timescale.

\subsection{Experimental Design}

We generate observations from a new reference run. We use a non-flat fixed bedrock whose elevation is defined by the equation

$$
\begin{aligned}
b(r)= & 1000 \mathrm{~m}-1400 \mathrm{~m} \cdot\left(\frac{r}{1000 \mathrm{~km}}\right)^{2} \\
& +700 \mathrm{~m} \cdot\left(\frac{r}{1000 \mathrm{~km}}\right)^{4}-120 \mathrm{~m} \cdot\left(\frac{r}{1000 \mathrm{~km}}\right)^{6} .
\end{aligned}
$$

The reference run is generated from a realistic initial state obtained with the following steps:

- Start with an ice sheet profile following Eq. (37) with $h_{0}=2000 \mathrm{~m}, r_{\max }=300 \mathrm{~km}$ and $n_{r}=21 \mathrm{compu}-$ tational mesh points evenly spaced between $\hat{r}_{1}=0$ and $\hat{r}_{n_{r}}=300 \mathrm{~km}$.

- Run the numerical model with a fixed climate forcing, as defined in Eq. (A4), where $T_{\text {clim }}=4{ }^{\circ} \mathrm{C}$ until it reaches the steady state (a 30000-year run with a $\Delta t=0.01$-year time step).

- From this steady state, run the numerical model with a linearly warming climate forcing from $T_{\mathrm{clim}}=4{ }^{\circ} \mathrm{C}$ with $\mathrm{d} T_{\text {clim }} / \mathrm{d} t=0.02{ }^{\circ} \mathrm{C} \mathrm{yr}^{-1}$ for an extra 100 years $(\Delta t=$ 


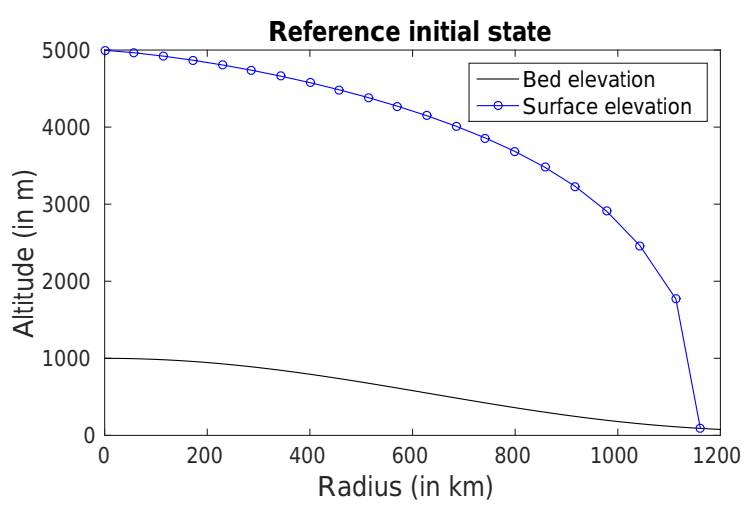

Figure 12. Initial state used to obtain a 20 -year reference run under a warming climate as detailed in Sect. 5.1 with $n_{r}=21$ grid points and a non-flat bed.

0.01 years). The state obtained at the end of the run is the initial state (see Fig. 12).

The reference is obtained by running the model over 20 years from the initial state with a time step $\Delta t=$ 0.01 years and the same linearly warming climate forcing as defined in Appendix A.2, with $T_{\text {clim }}=6^{\circ} \mathrm{C}$ at initial time $t=0$ years, and $T_{\text {clim }}=6.4^{\circ} \mathrm{C}$ at $t=20$ years (that is, $\left.T_{\text {clim }}=6+0.2 t\right)$. Over the 20-year run, the geometry of the ice sheet stays relatively similar to the geometry of the initial state due to the slow dynamics of the model. The ice sheet margin retreats from 1160.9 to $1158.6 \mathrm{~km}$ and the ice thickness at the ice divide increases by $1.5 \mathrm{~m}$.

We generate observations of surface elevation, surface ice velocity and the position of the ice sheet margin at times $t=$ $1,2, \ldots, 10$ years from the reference run. The observations of the surface are taken at each point including the margin with an added Gaussian noise (uncorrelated with standard deviation $\sigma_{s}^{o}=200 \mathrm{~m}$ ). The observations of the surface ice velocity are located at the midpoints between mesh points (so we have 20 observations of surface velocity). Observations are noised using a Gaussian law (standard deviation $\sigma_{u_{s}}^{o}=$ $30 \mathrm{~m} \mathrm{yr}^{-1}$, uncorrelated). For the position of the margin, the observational noise is sampled from $\mathcal{N}\left(0, \sigma_{r}^{o 2}\right)$ with $\sigma_{r}^{o}=$ $50 \mathrm{~km}$.

We compare the influence of the observations on the quality of the DA estimates and the subsequent forecasts for the 3D-Var and ETKF methods. Again, we focus on the two variables: the ice thickness at the ice divide at $r=0$ and the position of the ice sheet margin.

\subsection{Assimilating observations of surface elevation}

We begin by studying the performance of the DA schemes where we assimilate only observations of surface elevations.

For 3D-Var, the estimates are obtained using an initial background state defined as $\boldsymbol{x}^{b}=0.95 \boldsymbol{x}^{\mathrm{ref}}(0)$ with a $5 \%$ smaller extent than the reference state. The flow-dependent background error covariance matrix $\mathbf{B}$ is defined as in Eq. (28). The matrix $\mathbf{B}_{h}$ is defined as in Eq. (38) with a SOAR matrix for $\mathbf{C}_{h}\left(\sigma_{h}^{b}=200 \mathrm{~m}, L_{h}=240 \mathrm{~km}\right)$ and $\mathbf{B}_{r}$ is defined with a SOAR matrix for $\mathbf{C}_{r}\left(\sigma_{r}^{b}=60 \mathrm{~km}, L_{r}=240 \mathrm{~km}\right)$. The matrix $\mathbf{B}_{r h}$ is set to $\mathbf{0}$.

The ETKF uses an ensemble with 200 members. The initial ensemble is generated by adding to $\boldsymbol{x}^{b}$ a random noise drawn from the Gaussian law $\mathcal{N}(\boldsymbol{O}, \mathbf{B})$. The background covariance matrix $\mathbf{B}$ is defined as previously, except for $\mathbf{B}_{r}$ for which we still use a SOAR matrix for $\mathbf{C}_{r}\left(L_{r}=240 \mathrm{~km}\right)$ but with variances decreased near the ice divide following Eq. (41) $\left(\sigma_{r}^{b}=60 \mathrm{~km}\right.$ and $\left.\alpha=0.2\right)$. We tested different values for the inflation parameter $\lambda_{\text {infla }}$; the best results were obtained with $\lambda_{\text {infla }}=1.01$.

We first study the results obtained with the ETKF. At the end of the data assimilation window, $t=10$ years, the ice thickness profile is retrieved well everywhere by the mean of the ensemble and the reference profile is within the ensemble spread (see Fig. 13). We note that the estimate of the ice thickness at the ice divide is improved by the first analysis. After time $t=7$ years, however, the estimate is worsened by the analysis. This is because the ensemble spread is too small from that time onwards. This can be fixed by taking a larger inflation parameter $\lambda_{\text {infla }}$, but the estimates of other variables are then degraded. The estimated position (mean) of the margin at $t=10$ years is $1158.0 \mathrm{~km}$ with an ensemble standard deviation of $3.1 \mathrm{~km}$. In comparison to the reference value at that time, $r=1159.9 \mathrm{~km}$, we see that the ETKF with a large ensemble performs well. The quality of the estimates is also kept high during the forecast (from $t=10$ to $t=20$ years). For example, the absolute error on the position of the margin is kept below $2.5 \mathrm{~km}$ over this time window.

With respect to the covariance matrix, the estimates seem to show a similar behaviour to those of the experiment detailed in Sect. 4.4 using the ETKF where observations of ice thickness are assimilated (see Fig. 14), but with a larger correlation length scale. The similarity can be explained by the similarity of the construction of the initial ensemble (the same structure for the background covariance matrix $\mathbf{B}$ used to sample the Gaussian noise added to the background state) and by the similarity of the observation operators for ice thickness and surface elevation.

We now compare the ETKF with results obtained with 3D-Var. Absolute errors in the ice thickness at $r=0$ and in the position of the margins are displayed for both cases in Fig. 15. As in previous experiments, the ETKF performs better than 3D-Var. For example, the absolute error for the ice thickness at the ice divide stays below $60 \mathrm{~m}$ after $t=1$ year for the ETKF. By contrast, the absolute error for 3D-Var can be up to $125 \mathrm{~m}$. The same statement remains valid for the absolute error in the position of the margin, which stays below $8 \mathrm{~km}$ for the ETKF after $t=2$ years, yet can be up to $20 \mathrm{~km}$ for 3D-Var. We remark that, since the domain of the background state is smaller than that of the reference state, 
(a) Ice sheet geometry, $\mathrm{t}=\mathbf{1 0} \mathrm{yr}$

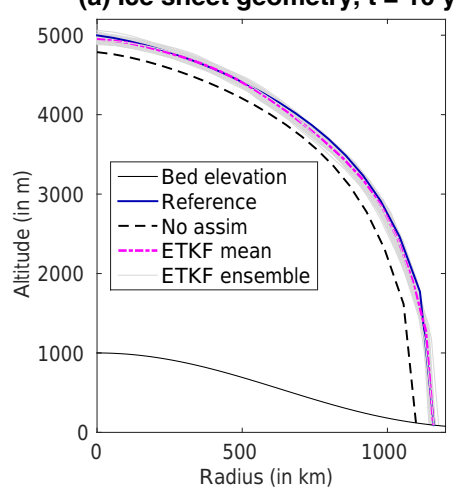

(b) Evolution ice thickness at $r=0$

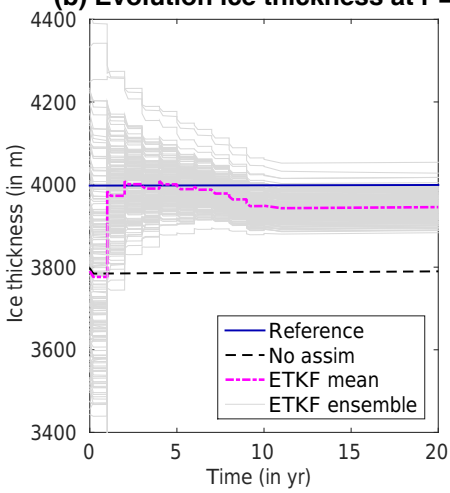

(c) Evolution position margin

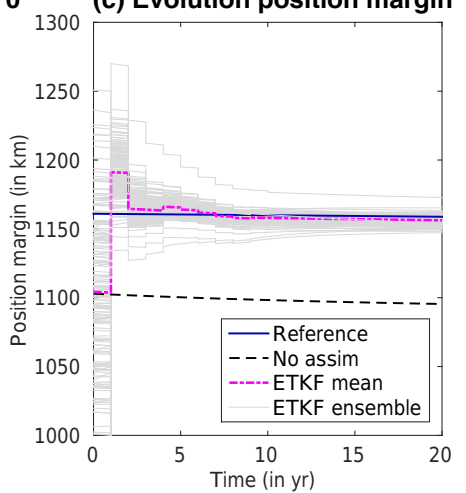

Figure 13. ETKF results for the advanced configuration where observations of surface elevation are assimilated over the first 10 years and a forecast is made for 10 further years. (a) ETKF analysis at time $t=10$ years compared with the reference. (b) Evolution of the ice thickness at $r=0$ with time. (c) Evolution of the position of the margin with time.
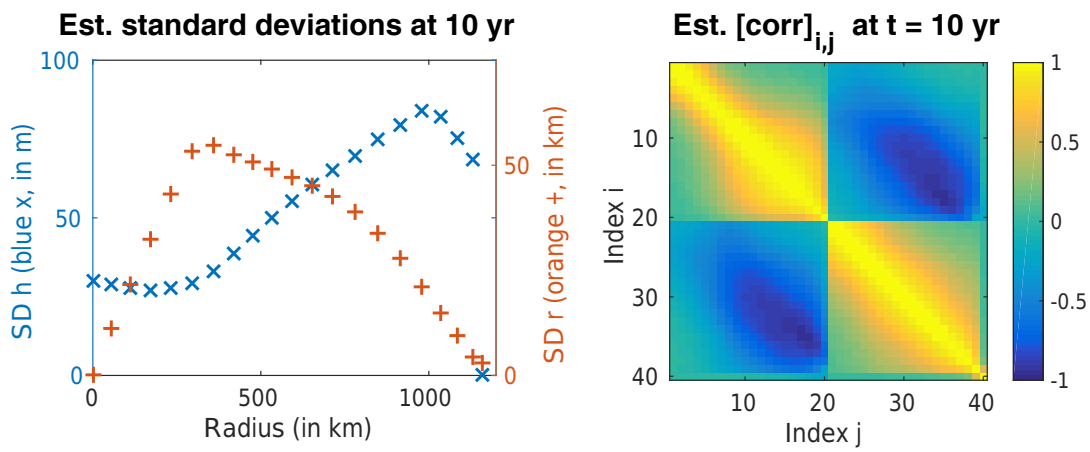

Figure 14. Standard deviations and correlation matrix Corr estimated from the analysis ensemble at time $t=10$ years in the advanced configuration where we observe surface elevation. The matrix Corr has the same structure as B defined by Eq. (28). Both standard deviations and correlation structures are similar to Fig. 8.

3D-Var does not assimilate all available data. Indeed, the algorithm cannot incorporate observations outside the background domain because of the form of the observation operator (see Eq. 32). This is not, however, the case for the ETKF, even if the ensemble mean has a smaller domain than the reference domain, since in this case there is at least one member of the ensemble with a bigger domain than that of the reference. At the end, both approaches show a similar accuracy in the forecast state after time $t=10$ years, showing again the efficiency of both DA schemes.

\subsection{Assimilating observations of surface velocity and position of the margin}

We now consider assimilating observations of surface ice velocity and the position of the margin (if we only assimilate observations of surface ice velocity, the problem is undetermined).

Again, we want to compare the accuracy of 3D-Var and the ETKF using this new set of observations. We use the same background state, the same structure for $\mathbf{B}$ and the same ini- tial ensemble as before. The observation operator for surface velocities is non-linear (see Eq. 35) and, even though the ensemble is large, inflation is necessary in this case. We take an inflation of $\lambda_{\text {infla }}=1.10$. If the inflation is taken any larger in this example, the ETKF analysis produces ensemble members with a non-ordered grid and the experiment cannot be pursued.

We first study the results obtained with the ETKF. At the end of the DA window, $t=10$ years, the ice thickness profile is retrieved well everywhere by the mean of the ensemble, except near the ice divide at $r=0$ (see Fig. 16). This is due to the relatively large uncertainty of surface velocity observations near the ice divide compared to the reference value at the same point (here $\sigma_{u_{s}}^{o}=30 \mathrm{~m} \mathrm{yr}^{-1}$ and the reference surface velocity near the ice divide is below $0.1 \mathrm{~m} \mathrm{yr}^{-1}$ ). The estimated (mean) position of the margin at $t=10$ years is $1144.7 \mathrm{~km}$ with an ensemble standard deviation of $12.1 \mathrm{~km}$. This is an absolute error of $15 \mathrm{~km}$, so it is worse than in the case where we observed the surface elevation, but assimilating these data still provides better estimates than those obtained with no assimilation. This comment remains valid for 

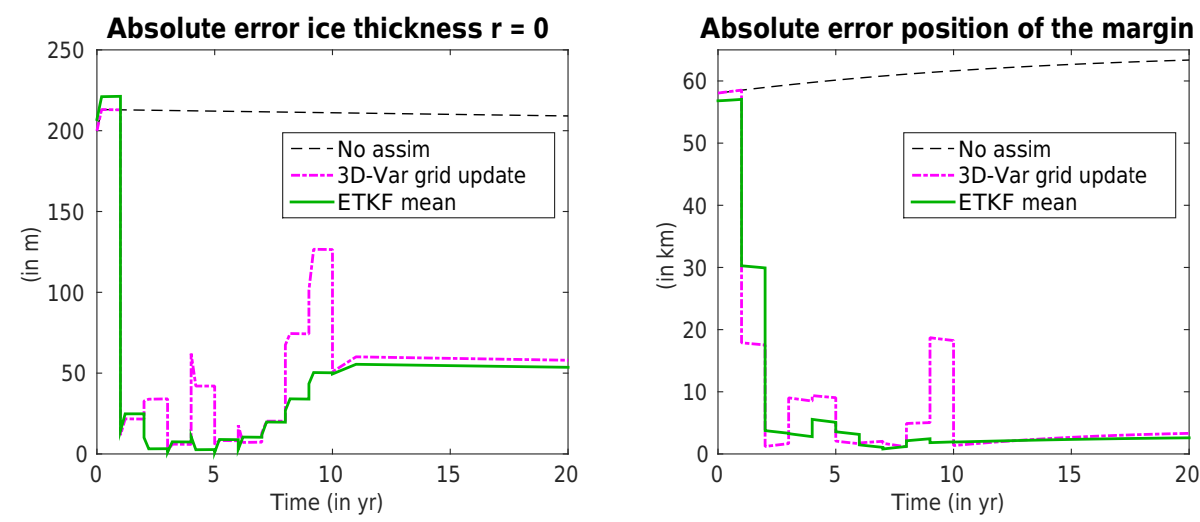

Figure 15. Evolution of the absolute error of the estimated ice thickness at $r=0$ and the estimated position of the margin in the advanced configuration where we assimilate surface elevations over the first 10 years. We compare the absolute errors obtained when we use 3D-Var with the correction of the position of grid nodes and when we use an ETKF. The ETKF performs better than the 3D-Var for both variables.

(a) Ice sheet geometry, $\mathrm{t}=10 \mathrm{yr}$

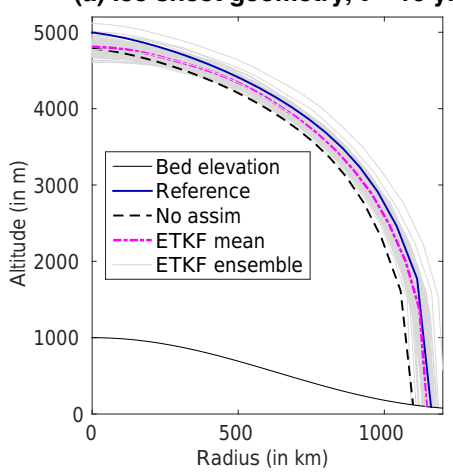

(b) Evolution ice thickness at $r=0$

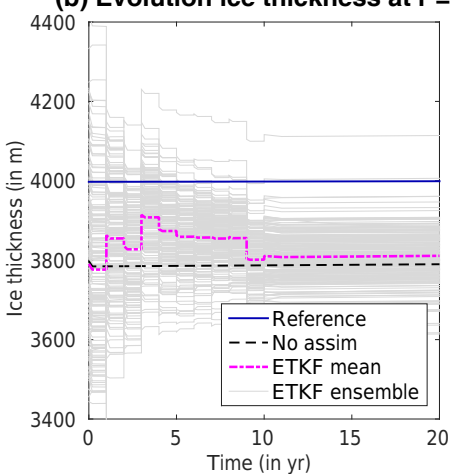

(c) Evolution position margin

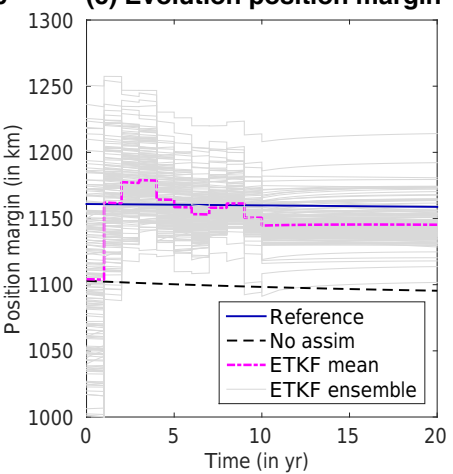

Figure 16. ETKF results for the advanced configuration where observations of surface ice velocity and the position of the margin are assimilated over the first 10 years and a forecast is made for the following 10 years. (a) ETKF analysis at time $t=10$ years compared with the reference. (b) Evolution of the ice thickness at $r=0$ with time. (c) Evolution of the position of the margin with time.

the forecasts obtained after $t=10$ years since estimates of the position of the margin are not degraded over the time window [10, 20 years].

Estimates of the standard deviations and covariances, as shown in Fig. 17, differ from those of the previous experiment (see Fig. 14 for comparison). The reduction in the standard deviation for ice thickness variables close to the ice divide is less significant than in the previous experiment. This is due to the relatively large uncertainty of surface velocity observations near the ice divide compared to the reference value at the same point. We remark that assimilating observations of surface ice velocity together with the position of the margin leads to an increased correlation length scale for ice thickness variables and to a smaller correlation length scale for node positions compared to the previous experiment. Finally, the cross-covariances have smaller anti-correlations and positive correlations appear between ice thickness variables in the interior of the ice sheet and between node positions close to the margin. These differ significantly from the case where we assimilate observations of surface elevation as a result of the difference in observation operators.

We finally compare the ETKF with results obtained with 3D-Var. Absolute errors in the ice thickness at $r=0$ and in the position of the margins are displayed for both cases in Fig. 18. As in previous experiments, the ETKF performs better than 3D-Var for the position of the margin, but 3D-Var gives better results for the ice thickness at $r=0$ and performs reasonably well overall in this non-linear context. The forecast trajectory of the margin after $t=10$ is improved by DA in both cases. This demonstrates again the robustness of our DA approach in the context of an ice sheet modelled with a moving point numerical model.

\section{Conclusion and prospects}

In this paper, we have adapted standard data assimilation techniques (a 3D-Var scheme and an ETKF) to estimate the state of a 1-D ice sheet model using a moving point method. This is done by including both ice thickness variables and 
Est. standard deviations at $10 \mathrm{yr}$

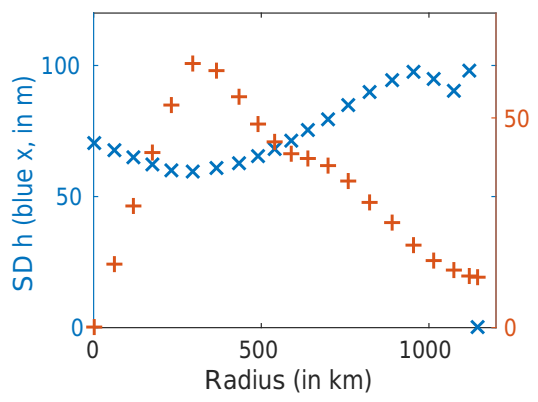

Est. [corr $_{\mathrm{i}, \mathrm{j}}$ at $\mathrm{t}=10 \mathrm{yr}$

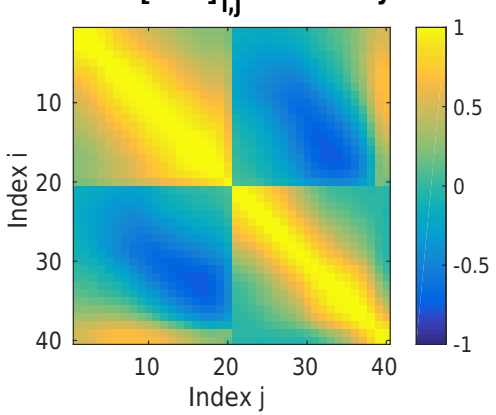

Figure 17. Standard deviations and correlation matrix Corr estimated from the analysis ensemble at time $t=10$ years in the advanced configuration where we observe surface ice velocity and the position of the margin. The matrix Corr has the same structure as $\mathbf{B}$ defined by Eq. (28). Both standard deviations and cross-correlation structures are different from those shown in Fig. 14.
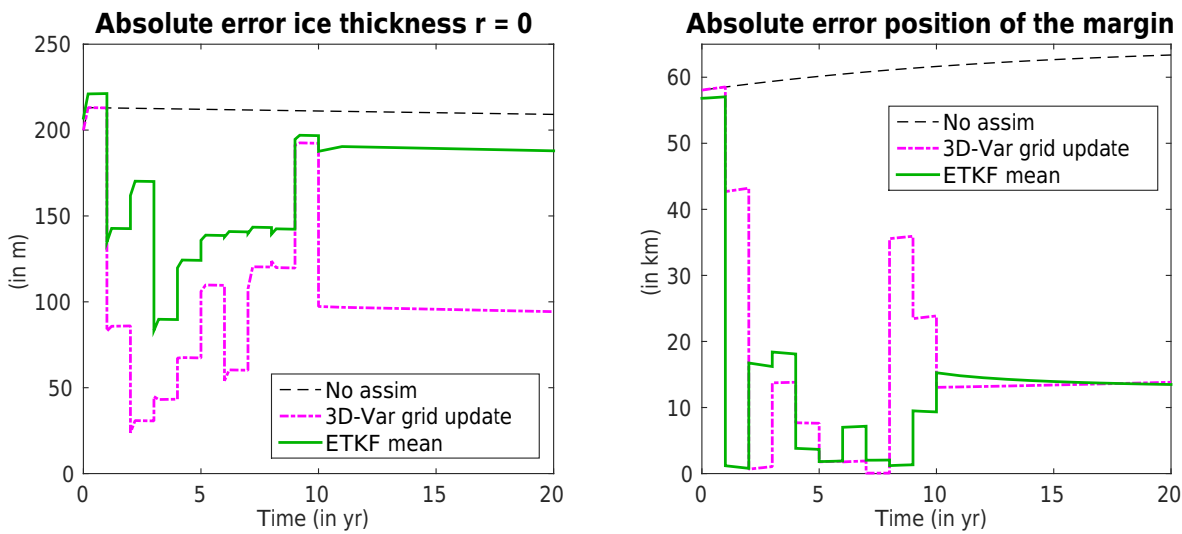

Figure 18. Evolution of the absolute error of the estimated ice thickness at $r=0$ and the estimated position of the margin when we observe surface ice velocities and the position of the margin in the advanced configuration. We compare the absolute errors obtained when we use 3D-Var with the correction of the grid-node positions and when we use an ETKF. The ETKF performs better than the 3D-Var with respect to the position of the margin, but 3D-Var gives better results for the ice thickness at $r=0$ in this case.

the location of mesh nodes in the state vector. The only requirement is to ensure that the update does not produce a non-ordered moving mesh. This can be achieved empirically either by using an appropriate flow-dependent background covariance matrix with large correlations between adjacent mesh points or by using an ensemble with the same properties. This combination has been validated with various twin experiments assimilating classical available observations for an ice sheet (ice thickness, surface elevation and surface ice velocity) and also observations of the position of the boundary. These twin experiments show the following:

- The form of the state vector allows the explicit tracking of boundary positions for moving boundary problems.

- This form also allows a straightforward and efficient assimilation of boundary positions (in this paper, the position of the margin).
- Assimilating spatially distributed observations gives better estimates if node locations are updated in the analysis step.

- 3D-Var can have issues with assimilating observations if they are located outside the forecast domain; the ETKF can overcome these issues if at least one member of the ensemble has its numerical domain large enough to include the location of these observations.

- ETKF tends to provide better estimates than 3DVar, mainly because of its capacity to provide flowdependent statistical estimates of the background error covariances, but 3D-Var still provides satisfactory estimates.

- ETKF provides not only good state estimates but also interesting information on the structure of the covariances; these are expected to be dominated by the statistics of the initial ensemble and the type of observations that are assimilated. 
Whilst this paper uses a particular moving mesh method for the 1-D numerical model, our approach can be extended to any 1-D moving boundary problem modelled with a moving mesh, assuming only that the ordering of the points must be maintained.

Moving mesh approaches are also suitable for modelling the evolution of 2-D moving boundary phenomena (Baines et al., 2009). The successful application of the moving mesh method to a 2-D model of an ice cap is presented in Partridge (2013). Initial results on the assimilation of observations of ice thickness into the 2-D ice cap model are also given in Partridge (2013). These results raise a number of issues concerning the approach needed for updating the nodal positions of the 2-D grid during the assimilation step. Research on these issues is ongoing.

Data availability. Data have been generated using the ice sheet model as described in the text and can be reproduced by the reader using the algorithm given in Bonan et al. (2016). The ETKF used in this paper follows the implementation of Hunt et al. (2007). The 3D-Var method and the design of each experiment are fully detailed in the paper. 


\section{Appendix A: Surface mass balances}

\section{A1 EISMINT surface mass balance}

For the twin experiments performed in Sect. 4, we use the simple constant-in-time surface mass balance employed in the moving margin experiments of the EISMINT intercomparison project (Huybrechts et al., 1996):

$m(r)=\min \left(0.5 \mathrm{~m} \mathrm{yr}^{-1}, 10^{-2} \mathrm{myr}^{-1} \mathrm{~km}^{-1} \cdot(450 \mathrm{~km}-r)\right)$.

\section{A2 Parameterised surface mass balance with feedback loop}

For the twin experiments performed in Sect. 5, we use a more complex surface mass balance parameterised as a function of the surface atmospheric temperature $T_{s}(t, r)$. This simple parameterisation was used in Bonan et al. (2014) in the context of ice sheet model initialisation but with a fixed-grid model. The values of the different parameters involved in this parameterisation are given in Table A1. The surface mass balance is the sum of positive accumulation Acc (snow precipitation) and negative ablation $\mathrm{Abl}$ (melting) parameterised in Eqs. (A2) and (A3).

$$
\begin{aligned}
& \operatorname{Acc}(t, r)=\operatorname{Acc}_{0} e^{c_{0} T_{s}} \\
& \operatorname{Abl}(t, r)= \begin{cases}\operatorname{Abl}_{0}\left(\frac{T_{s}-T_{0}}{T_{0}}\right)^{2} & \text { if } T_{s}>T_{0} \\
0 & \text { otherwise }\end{cases}
\end{aligned}
$$

The surface temperature depends on the altitude of the surface $s$, the distance from the origin and a climate temperature $T_{\text {clim }}(t)$ evolving in time according the relation

$T_{s}(t, r)=T_{\mathrm{clim}}(t)+\lambda r+\gamma s(t, r)$.

This parameterisation aims to reproduce qualitatively a typical surface mass balance over an ice sheet and to include feedbacks associated with the evolution of the geometry.

Table A1. List of parameter values used for the parameterised sur-

\begin{tabular}{|c|c|c|}
\hline Parameter & & Value \\
\hline $\mathrm{Acc}_{0}$ & rate of accumulation & $6 \mathrm{myr}^{-1}$ \\
\hline $\mathrm{Abl}_{0}$ & rate of ablation & $-5 \mathrm{~m} \mathrm{yr}^{-1}$ \\
\hline$T_{0}$ & $\begin{array}{l}\text { minimum temperature for } \\
\text { ablation }\end{array}$ & $-6^{\circ} \mathrm{C}$ \\
\hline$c_{0}$ & $\begin{array}{l}\text { coefficient exponential law } \\
\text { for accumulation }\end{array}$ & $0.115^{\circ} \mathrm{C}^{-1}$ \\
\hline$\lambda$ & $\begin{array}{l}\text { longitudinal gradient of } \\
\text { surface temperature }\end{array}$ & $\frac{1}{111000}{ }^{\circ} \mathrm{Cm}^{-1}$ \\
\hline$\gamma$ & $\begin{array}{l}\text { vertical gradient of } \\
\text { surface temperature }\end{array}$ & $-0.0063^{\circ} \mathrm{C} \mathrm{m}^{-1}$ \\
\hline
\end{tabular}
face mass balance. 
Competing interests. The authors declare that they have no conflict of interest.

Acknowledgements. This research was funded in part by the Natural Environmental Research Council National Centre for Earth Observation (NCEO) and the European Space Agency (ESA).

Edited by: Olivier Talagrand

Reviewed by: two anonymous referees

\section{References}

Anderson, J. L. and Anderson, S. L.: A Monte Carlo implementation of the nonlinear filtering problem to produce ensemble assimilations and forecasts, Mon. Weather Rev., 127, 2741-2758, https://doi.org/10.1175/15200493(1999)127<2741:AMCIOT>2.0.CO;2, 1999.

Baines, M. J., Hubbard, M. E., and Jimack, P. K.: A moving mesh finite element algorithm for the adaptive solution of time-dependent partial differential equations with moving boundaries, Appl. Numer. Math., 54, 450-469, https://doi.org/10.1016/j.apnum.2004.09.013, 2005.

Baines, M. J., Hubbard, M. E., Jimack, P. K., and Mahmood, R.: A moving-mesh finite element method and its application to the numerical solution of phase-change problems, Commun. Comput. Phys., 6, 595-624, 2009.

Baines, M. J., Hubbard, M. E., and Jimack, P. K.: Velocitybased moving mesh methods for nonlinear partial differential equations, Commun. Comput. Phys., 10, 509-576, https://doi.org/10.4208/cicp.201010.040511a, 2011.

Berger, M. J. and Oliger, J.: Adaptive mesh refinement for hyperbolic partial differential equations, J. Comput. Physics, 53, 484 $512,1984$.

Bishop, C. H., Etherton, B. J., and Majumdar, S. J.: Adaptive sampling with the Ensemble Transform Kalman Filter. Part I: Theoretical aspects, Mon. Weather Rev., 129, 420-436, https://doi.org/10.1175/15200493(2001)129<0420:ASWTET>2.0.CO;2, 2001.

Blayo, E., Bocquet, M., Cosme, E., and Cugliandolo, L. F.: Advanced Data Assimilation for Geosciences, Lecture Notes of the Les Houches School of Physics: Special Issue, June 2012, Oxford University Press, Oxford, UK, 2014.

Bonan, B., Nodet, M., Ritz, C., and Peyaud, V.: An ETKF approach for initial state and parameter estimation in ice sheet modelling, Nonlin. Processes Geophys., 21, 569-582, https://doi.org/10.5194/npg-21-569-2014, 2014.

Bonan, B., Baines, M. J., Nichols, N. K., and Partridge, D.: A moving-point approach to model shallow ice sheets: a study case with radially symmetrical ice sheets, The Cryosphere, 10, 1-14, https://doi.org/10.5194/tc-10-1-2016, 2016.

Budd, C. J., Huang, W., and Russell, R. D.: Adaptivity with moving grids, Acta Numerica, 18, 111-241, https://doi.org/10.1017/S0962492906400015, 2009.

Cao, W., Huang, W., and Russell, R. D.: Approaches for generating moving adaptive meshes: location versus velocity, Appl. Numer. Math., 47, 121-138, https://doi.org/10.1016/S01689274(03)00061-8, 2003
Church, J. A., Clark, P. U., Cazenave, A., Gregory, J. M., Jevrejeva, S., Leverman, A., Merrifield, M. A., Milne, G. A., Nerem, R. S., Nunn, P. D., Payne, A. J., Pfeffer, W. T., Stammer, D., and Unnikrishnan, A. S.: Sea level change, in: Climate Change 2013: The Physical Science Basis, Contribution of Working Group I to the Fifth Assessment Report of the Intergovernmental Panel on Climate Change, edited by: Stocker, T. F., Qin, D., Plattner, G.-K., Tignor, M., Allen, S. K., Boschung, J., Nauels, A., Xia Y., Bex, V., and Midgley, P. M., , Cambridge University Press, Cambridge, UK, New York, NY, USA, 1137-1216, 2013.

Cornford, S. L., Martin, D. F., Graves, D. T., Ranken, D. F., Le Brocq, A. M., Gladstone, R. M., Payne, A. J., Ng, E. G., and Lipscomb, W. H.: Adaptive mesh, finite volume modeling of marine ice sheets, J. Comput. Physics, 232, 529-549, https://doi.org/10.1016/j.jcp.2012.08.037, 2013.

Cornford, S. L., Martin, D. F., Lee, V., Payne, A., and Ng, E. G.: Adaptive mesh refinement versus subgrid friction interpolation in simulations of Antarctic ice dynamics, Ann. Glaciol., 57, 1-9, https://doi.org/10.1017/aog.2016.13, 2016.

Dyke, A. S. and Prest, V. K.: Late Wisconsinan and Holocene Retreat of the Laurentide Ice Sheet. Scale 1:5000 000, Map 1702A, Geological Survey of Canada, Ottawa, Ontario, Canada, 1987.

Evensen, G.: Sequential data assimilation with a nonlinear quasigeostrophic model using Monte Carlo methods to forecast error statistics, J. Geophys. Res.-Oceans, 99, 10143-10162, https://doi.org/10.1029/94JC00572, 1994.

Gladstone, V., Lee, A., Vieli, A., and Payne, A. J.: Grounding line migration in an adaptive mesh ice sheet model, J. Geophys. Res.-Earth, 115, F04014, https://doi.org/10.1029/2009JF001615, 2010.

Haben, S. A., Lawless, A. S., and Nichols, N. K.: Conditioning of incremental variational data assimilation, with application to the Met Office system, Tellus A, 63, 782-792, 2011.

Hunt, B. R., Kostelich, E. J., and Szunyogh, I.: Efficient data assimilation for spatiotemporal chaos: A local ensemble transform Kalman filter, Physica D, 230, 112-126, https://doi.org/10.1016/j.physd.2006.11.008, 2007.

Hutter, K.: Theoretical Glaciology, D. Reidel, Dordrecht, the Netherlands, 1983.

Huybrechts, P., Payne, A. J., and The EISMINT Intercomparison Group: The EISMINT benchmarks for testing ice-sheet models, Ann. Glaciol., 23, 1-12, 1996.

Lahoz, W., Khattatov, B., and Menard, R. E.: Data assimilation: making sense of observations, Springer-Verlag, Berlin, Germany, https://doi.org/10.1007/978-3-540-74703-1, 2010.

Lecavalier, B. S., Milne, G. A., Simpson, M. J. R., Wake, L., Huybrechts, P., Tarasov, L., Kjeldsen, K. K., Funder, S., Long, A. J., Woodroffe, S., Dyke, A. S., and Larsen, N. K.: A model of Greenland ice sheet deglaciation constrained by observations of relative sea level and ice extent, Quaternary Sci. Rev., 102, 5484, https://doi.org/10.1016/j.quascirev.2014.07.018, 2014.

Lee, T. E., Baines, M. J., Langdon, S., and Tindall, M. J.: A moving mesh approach for modelling avascular tumour growth, Appl. Numer. Math., 72, 99-114, https://doi.org/10.1016/j.apnum.2013.06.001, 2013.

Lee, T. E., Baines, M. J., and Langdon, S.: A finite difference moving mesh method based on conservation for moving boundary problems, J. Comput. Appl. Math., 288, 1-17, https://doi.org/10.1016/j.cam.2015.03.032, 2015. 
Li, Y., Jeong, D., and Kim, J.: Adaptive mesh refinement for simulation of thin film flows, Meccanica, 49, 239-252, https://doi.org/10.1007/s11012-013-9788-6, 2014.

Lorenc, A. C.: Analysis methods for numerical weather prediction, Q. J. Roy. Meteor. Soc., 112, 1177-1194, https://doi.org/10.1002/qj.49711247414, 1986.

Lukyanov, A. V., Sushchikh, M. M., Baines, M. J., and Theofanous, T. G.: Superfast nonlinear diffusion: Capillary transport in particulate porous media, Phys. Rev. Lett., 109, 214501, https://doi.org/10.1103/PhysRevLett.109.214501, 2012.

Mathiot, P., König Beatty, C., Fichefet, T., Goosse, H., Massonnet, F., and Vancoppenolle, M.: Better constraints on the sea-ice state using global sea-ice data assimilation, Geosci. Model Dev., 5, 1501-1515, https://doi.org/10.5194/gmd-5-1501-2012, 2012.

Nichols, N. K.: Mathematical concepts of data assimilation, in: Data assimilation: making sense of observations, edited by: Lahoz, W., Khattatov, B. and Menard, R., Springer-Verlag, Berlin, Germany, 13-40, 2010.

Partridge, D.: Numerical modelling of glaciers: moving meshes and data assimilation, PhD thesis, University of Reading, Reading, Berks, United Kingdom, available at: http://www.reading. ac.uk/web/FILES/maths/DP_PhDThesis.pdf (last access: $28 \mathrm{Au}-$ gust 2017), 2013.
Sarahs, N.: Similarity, Mass Conservation, and the $\mathrm{Nu}-$ merical Simulation of a Simplified Glacier Equation, SIAM Undergraduate Research Online, 9, S014019, https://doi.org/10.1137/15S014198, 2016.

Vaughan, D. G., Comiso, J. C., Allison, I., Carrasco, J., Kaser, G., Kwok, R., Mote, P., Murray, T., Paul, F., Ren, J., Rignot, E., Solomina, O., Steffen, K., and Zhang, T.: Observations: Cryosphere, in: Climate Change 2013: The Physical Science Basis, Contribution of Working Group I to the Fifth Assessment Report of the Intergovernmental Panel on Climate Change, edited by: Stocker, T. F., Qin, D., Plattner, G.-K., Tignor, M., Allen, S. K., Boschung, J., Nauels, A., Xia, Y., Bex, V., and Midgley, P. M., Cambridge University Press, Cambridge, UK, New York, NY, USA, 317-382, 2013. 